Article

\title{
Fuzzy Decision Support Modeling for Hydrogen Power Plant Selection Based on Single Valued Neutrosophic Sine Trigonometric Aggregation Operators
}

\author{
Shahzaib Ashraf ${ }^{1}{ }^{(}$, Saleem Abdullah ${ }^{1}{ }^{(}$, Shouzhen Zeng ${ }^{2,3} \mathbb{C}^{-}$, Huanhuan Jin ${ }^{4, *}$ and Fazal \\ Ghani $^{1}$ \\ 1 Department of Mathematics, Abdul Wali Khan University, Mardan 23200, Pakistan; \\ shahzaibashraf@awkum.edu.pk (S.A.); saleemabdullah@awkum.edu.pk (S.A.); \\ fghanimath@awkum.edu.pk (F.G.) \\ 2 School of Business, Ningbo University, Ningbo 315100, China; zszzx1@163.com \\ 3 School of Management, Fudan University, Shanghai 200433, China \\ 4 College of Statistics and Mathematics, Zhejiang Gongshang University, Hangzhou 310018, China \\ * Correspondence: Jinhh06@163.com
}

Received: 14 January 2020; Accepted: 7 February 2020; Published: 19 February 2020

\begin{abstract}
In recent decades, there has been a massive growth towards the prime interest of the hydrogen energy industry in automobile transportation fuel. Hydrogen is the most plentiful component and a perfect carrier of energy. Generally, evaluating a suitable hydrogen power plant site is a complex selection of multi-criteria decision-making (MCDM) problem concerning proper location assessment based on numerous essential criteria, the decision-makers expert opinion, and other qualitative/quantitative aspects. This paper presents the novel single-valued neutrosophic (SVN) multi-attribute decision-making method to help decision-makers choose the optimal hydrogen power plant site. At first, novel operating laws based on sine trigonometric function for single-valued neutrosophic sets (SVNSs) are introduced. The well-known sine trigonometry function preserves the periodicity and symmetric in nature about the origin, and therefore it satisfies the decision-maker preferences over the multi-time phase parameters. In conjunction with these properties and laws, we define several new aggregation operators (AOs), called SVN weighted averaging and geometric operators, to aggregate SVNSs. Subsequently, on the basis of the proposed AOs, we introduce decision-making technique for addressing multi-attribute decision-making (MADM) problems and provide a numerical illustration of the hydrogen power plant selection problem for validation. A detailed comparative analysis, including a sensitivity analysis, was carried out to improve the understanding and clarity of the proposed methodologies in view of the existing literature on MADM problems.
\end{abstract}

Keywords: single-valued neutrosophic sets; sine trigonometric operational laws; sine trigonometric aggregation operators; decision-making technique; hydrogen power plant selection problem

\section{Introduction}

Fossil fuels and renewable energy are the most important natural resources for the social and economic growth of a country. Invariably and exceptionally, it is clearly observed that energy demand is increasing significantly over time throughout the world. The major dependence on fossil fuels leads directly to carbon dioxide emissions that harm the environment and also rapidly exhaust the natural stock. The implementation of the electrification technique decreased the emission factor dramatically, but could not ultimately be considered a viable solution. However, hydrogen energy, wind power, 
biomass fertility, biofuel, solar energy, geothermal, etc. are the sustainable energy sources that can be misused and used in practical purposes that have been differentiated up to this point.

In the current scenario, the viability in terms of technology, economic efficiency and environment give rise to the selection of hydrogen energy as a new kind of renewable energy source. Significant advantageous features of hydrogen are as follows, it is a extreme heat-burning gas and its chemical composition is free from elements which on combustion releases toxic gases e.g., $\mathrm{CO}_{2}, \mathrm{SO}_{2}$, and $\mathrm{NO}_{2}$, whereas hydrogen releases only water upon combustion. As hydrogen can be obtained from water (electrolysis) and solar energy (solar hydrogen), we can have an ample and endless source of hydrogen energy for the society and its need. Therefore, the consideration of hydrogen energy is supposed to be a kind of clean renewable energy having perfectly zero emissions for the future prospects and it has received due attention of the researchers in recent past [1]. Various researchers dealt with issues of felicitating the hydrogen energy for the energy balance [2]. For the sake of electricity production, alternative energy sources such as hydrogenated fuels have also been utilized in [3]. Juste [4] experimented with hydrogen injection as an augmented fuel and investigated the gas turbine combustion chambers.

Over the past few decades, numerous researchers and decision-makers have focused almost entirely on renewable energy/technology selection issues, particularly on hydrogen power, which has always been a major task. The task of choosing the right and most appropriate site for such sustainable energy comprises of demographic view point, socio-economic factor and infrastructure. The decision-making algorithms certainly enhance the capabilities of the experts/decision-makers to moderate the content of decisions in terms of their rationality and efficiency in a better sense. The process of site selection for hydrogen power plant can be modeled as a multi-attribute decision-making (MADM) problem as various available inter-conflicting attributes must be explored.

There are several challenges in decision-making due to uncertainty. Zadeh [5] developed the concept of a fuzzy set (FS) in 1965 to address the uncertainty in decision-making problems (DMPs). FSs can describe fuzzy information in real-life, and analyze a certain imprecise phenomenon. Since then, researches on FSs have emerged in large numbers, like FSs for decision-making [6,7]. In 1983, Atanassove [8] proposed the intuitionistic fuzzy set (IFS) to extend FSs. IFS contains positive and negative membership grades that meet the sum of two grades being less than or equal to 1 . Thereby, ushering a new era for fuzzy mathematics, and many studies such as aggregation operator [9-11] have been completed. Based on IFS, to break its constraint, Yager [12] given a notion of generalized orthopair fuzzy set, i.e., q-rung orthopair fuzzy set (q-ROFS). There are also a positive and negative membership grades included in q-ROFS, but they satisfy the q-th power of membership grades makes a result which is less than or equal to 1 . From the respective of available universe, it is obvious q-ROFSs can describe more fuzzy data than IFSs, thus many researches concerning q-ROFS [13-18] appeared.

Both the IF set and the PyF set addressed just two classes, i.e., "yes" and "no", but in the case of selection we have three types of responses, e.g., "yes", "no", and "neutral", and the complicated answer is "refusal". To overcome this business, Cuong [19] implemented a novel concept of picture fuzzy set (PFS), dignifying the positive, neutral and negative membership grades with the condition that sum of its membership grades be less or equal to 1 . Since then, researches on PFSs have emerged in large numbers, like decision-making techniques under picture fuzzy information are discussed in [20-27]. However, in some uncertain environments, the sum of positive, neutral and negative membership grades may be greater than 1, which is not suitable for PFS. In view of this, the spherical fuzzy set (SFS) [28] proposed by Ashraf and Abdullah relaxes the condition to allow the sum of the membership grades to be greater than 1 , whose sum of squares is less than or equal to 1 .

Since Ashraf and Abdullah proposed the spherical fuzzy set theory, many scholars have introduced various aggregation methods for handling the spherical fuzzy data, which enriched the theory and application of SFS. Ashraf et al. [29] presented the arithmetic/geometric aggregation operations over the spherical fuzzy sets. Also, Ashraf et al. [30] presented the concept of the spherical fuzzy Dombi aggregation operators under spherical fuzzy information. Jin et al. [31] developed the 
logarithmic-based aggregation operators for spherical fuzzy numbers to deal with uncertainty in decision-making problems. Jin et al. [32] proposed the linguistic spherical fuzzy aggregation operators under SF information. Rafiq et al. [33] proposed the decision-making technique based on the cosine similarity measures under SF information. Ashraf et al. [34] presented the spherical distance measure based decision-making technique under spherical fuzzy environments. Ashraf et al. [35] introduced the spherical fuzzy set representation of spherical fuzzy t-norm and t-conorm and discussed the TOPSIS-based decision-making technique under SF information. Zeng et al. [36] introduced the spherical fuzzy rough set based TOPSIS approach to deal the uncertainty in the form of spherical fuzzy sets. Ashraf et al. [37] presented the GRA technique user spherical linguistics fuzzy information by utilizing the concept of Choquet integral.

Smarandache [38] presented a novel set called neutrosophic set (NS), which is consist of a truth-membership, an indeterminacy-membership and a falsity-membership functions. Each membership function is a non-standard subset of a non-standard interval. Three membership functions of NS are mutually independent, therefore NS can be utilized to deal with more varied fuzzy events than previous fuzzy sets. Wang et al. [39] presented the single-valued neutrosophic set (SVNS). Due to the superiority of SVNS, there are many researches associated with it, such as Ye [40] that introduced the correlation coefficient of SVNSs. Liu et al. [41] presented the 2-tuple linguistic Dombi power Heronian mean AOs under SVN information. Liu et al. [42] presented the power muirhead mean AOs under SVN information and discussed their application in decision-making problem. liu et al. [43] established the power Heronian AOs under linguistic neutrosophic information and discussed their application in decision-making. Liu et al. [44] presented the group decision-making technique under hesitant interval neutrosophic uncertain linguistic information. Ye [45] introduced the cross-entropy under SVN information and also discussed their application in DMPs. Subsequently, some aggregation operators were studied, like [46-48]. From many theories and applications of SVNS, its applicability has been realized. For more study, we refer to [49-53].

It is understood that the laws of the operation play a key role model for any aggregation process. In that direction, Ye [54] defined the exponential operational laws (EOLs) for interval neutrosophic set and bases as real numbers. However, in terms of SVNSs, Garg [55] and Ashraf et al. [56] defined the logarithm operational laws (LOLs) under the SVNSs. Another important function apart from these exponential and logarithmic mathematical functions is the sine trigonometry (ST) function, which plays a dominant role during the aggregation of data. The major advantages of this function are its periodicity and that it is symmetric about the origin, and therefore it satisfies the decision-maker preferences over the multi-time phase parameters. In this manner, by keeping in mind the advantages and usefulness of ST function, there is a need to build up some new ST operational laws (STOLs) for SVNSs and SVNNs and studies their behavior. As a consequence, the aim of the paper is to design some new operational laws for SVNSs by challenging the above mentioned points and therefore presented the MADM algorithm to managing the evaluation information for SVNSs.

The rest of this paper is arranged as follows. Section 2 presents some knowledge related to FSs, IFSs, PyFSs, PFSs, SFSs, and SVNSs. Section 3 gave some novel sine trigonometric operational laws for SVNNs. In Section 4, proposed the sine trigonometric operational laws based aggregation operators under single-valued neutrosophic information, together with related proof on its properties. Backbone of this work is the novel decision-making technique to deal the uncertainty in decision-making problems to sort out the finest alternative according to list of attributes is proposed in Section 5 . Section 6 reports a numerical example on hydrogen power plant selection problem is provided to illustrate the feasibility of the proposed method, and some comparative analyses are conducted. The paper ends with some conclusions in Section 7.

\section{Preliminaries}

Some fundamental notions of FS, IFS, PFS, SFS, and SVNS have been explored here in this section. 


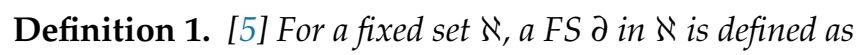

$$
\partial=\left\{\left\langle\hbar, \beth_{\partial}(\hbar)\right\rangle \mid \hbar \in \aleph\right\},
$$

for each $\hbar \in \aleph$, the positive membership grade $\beth_{\partial}: \aleph \rightarrow \Theta$ specifies the degree to which the element $\hbar \in \aleph$, where $\Theta=[0,1]$ is the unit interval.

Definition 2. [8] For a fixed set $\aleph$, an IFS $\partial$ in $\aleph$ is defined as

$$
\partial=\left\{\left\langle\hbar, \beth_{\partial}(\hbar), \beth_{\partial}(\hbar)\right\rangle \mid \hbar \in \aleph\right\},
$$

for each $\hbar \in \aleph$, the positive, negative membership grades, $\beth_{\partial}: \aleph \rightarrow \Theta$ and $\beth_{\partial}: \aleph \rightarrow \Theta$, respectively, of the element $\hbar$ to the IFS $\partial$, where $\Theta=[0,1]$ is the unit interval. Furthermore, it is required that $0 \leq$ $\beth_{\partial}(\hbar)+\beth_{\partial}(\hbar) \leq 1$, for each $\hbar \in \aleph$.

Definition 3. [19] For a fixed set $\aleph$, a PFS $\partial$ in $\aleph$ is defined as

$$
\left.\partial=\left\{\left\langle\hbar, \beth_{\partial}(\hbar),\right\rceil_{\partial}(\hbar), \beth_{\partial}(\hbar)\right\rangle \mid \hbar \in \aleph\right\},
$$

for each $\hbar \in \aleph$, the positive, neutral, and negative membership grades $\left.\beth_{\partial}: \aleph \rightarrow \Theta,\right\rceil_{\partial}: \aleph \rightarrow \Theta$ and $\mathrm{J}_{\partial}: \aleph \rightarrow \Theta$, respectively, of the element $\hbar$ to the PFS $\partial$, where $\Theta=[0,1]$ is the unit interval. Furthermore, it is required that $\left.0 \leq \beth_{\partial}(\hbar)+\right\rceil_{\partial}(\hbar)+\beth_{\partial}(\hbar) \leq 1$, for each $\hbar \in \aleph$.

Definition 4. [28] For a fixed set $\aleph$, a SFS $\partial$ in $\aleph$ is defined as

$$
\partial=\left\{\left\langle\hbar, \beth_{\partial}(\hbar), \nearrow_{\partial}(\hbar), \beth_{\partial}(\hbar)\right\rangle \mid \hbar \in \aleph\right\},
$$

for each $\hbar \in \aleph$, the positive, neutral, and negative membership grades $\left.\beth_{\partial}: \aleph \rightarrow \Theta,\right\rceil_{\partial}: \aleph \rightarrow \Theta$ and $J_{\partial}: \aleph \rightarrow \Theta$ respectively, of the element $\hbar$ to the SFS $\partial$, where $\Theta=[0,1]$ is the unit interval. Furthermore, it is required that $\left.0 \leq \beth_{\partial}^{2}(\hbar)+\right\rceil_{\partial}^{2}(\hbar)+\beth_{\partial}^{2}(\hbar) \leq 1$, for each $\hbar \in \aleph$.

Definition 5. [38] For a fixed set $\aleph$, a neutrosophic set $\partial$ in $\aleph$ is defined as

$$
\left.\partial=\left\{\left\langle\hbar, \beth_{\partial}(\hbar),\right\rceil_{\partial}(\hbar), \beth_{\partial}(\hbar)\right\rangle \mid \hbar \in \aleph\right\},
$$

for each $\hbar \in \aleph$, the truth, indeterminacy, and falsity membership grades $\left.\beth_{\partial}: \aleph \rightarrow \Theta,\right\rceil_{\partial}: \aleph \rightarrow \Theta$, and $\mathrm{J}_{\partial}: \aleph \rightarrow \Theta$, respectively, of the element $\hbar$ to the neutrosophic set $\partial$, where $\left.\Theta=\right] 0^{-}, 1^{+}[$. Furthermore, it is required that $\left.0^{-} \leq \beth_{\partial}(\hbar)+\right\rceil_{\partial}(\hbar)+\beth_{\partial}(\hbar) \leq 3^{+}$, for each $\hbar \in \aleph$.

Definition 6. [39] For a fixed set $\aleph$, a single-valued neutrosophic set (SVNS) $\partial$ in $\aleph$ is defined as

$$
\partial=\left\{\left\langle\hbar, \beth_{\partial}(\hbar), \nearrow_{\partial}(\hbar), \beth_{\partial}(\hbar)\right\rangle \mid \hbar \in \aleph\right\},
$$

for each $\hbar \in \aleph$, the truth, indeterminacy, and falsity membership grades $\left.\beth_{\partial}: \aleph \rightarrow \Theta,\right\rceil_{\partial}: \aleph \rightarrow \Theta$ and $J_{\partial}: \aleph \rightarrow \Theta$, respectively, of the element $\hbar$ to the neutrosophic set $\partial$, where $\Theta=[0,1]$ is the unit interval. Furthermore, it is required that $\left.0 \leq \beth_{\partial}(\hbar)+\right\rceil_{\partial}(\hbar)+\beth_{\partial}(\hbar) \leq 3$, for each $\hbar \in \aleph$.

In simplicity, the triplet $\left.\partial=\left\{\beth_{\partial},\right\urcorner_{\partial}, \beth_{\partial}\right\}$ called single-valued neutrosophic number (SVNN) in whole study and collection of SVNNs denoted by SVNN ( $)$.

Wang et al. [39], Ye [57], and Zhang \& Bo [58] introduced the basic operational laws of SVNNs, which are as follows. 
Definition 7. [58] Let $\left.\partial_{1}=\left\{\beth_{\partial_{1}},\right\rceil_{\partial_{1}}, \beth_{\partial_{1}}\right\}$ and $\left.\partial_{2}=\left\{\beth_{\partial_{2}},\right\urcorner_{\partial_{2}}, \beth_{\partial_{2}}\right\} \in S V N N(\aleph)$. than,

(1) $\partial_{1} \subseteq \partial_{2}$ if and only if $\left.\left.\beth_{\partial_{1}} \leq \beth_{\partial_{2}},\right\rceil_{\partial_{1}} \geq\right\rceil_{\partial_{2}}$ and $\beth_{\partial_{1}} \geq \beth_{\partial_{2}}$ for each $\hbar \in \aleph$.

(2) $\partial_{1}=\partial_{2}$ if and only if $\partial_{1} \subseteq \partial_{2}$ and $\partial_{2} \subseteq \partial_{1}$.

(3) $\left.\left.\partial_{1} \cap \partial_{2}=\left\{\inf \left(\beth_{\partial_{1}}, \beth_{\partial_{2}}\right), \sup (\rceil_{\partial_{1}},\right\rceil_{\partial_{2}}\right), \sup \left(\beth_{\partial_{1}}, \beth_{\partial_{2}}\right)\right\}$,

(4) $\left.\left.\partial_{1} \cup \partial_{2}=\left\{\sup \left(\beth_{\partial_{1}}, \beth_{\partial_{2}}\right), \inf (\rceil_{\partial_{1}},\right\rceil_{\partial_{2}}\right), \inf \left(\beth_{\partial_{1}}, \beth_{\partial_{2}}\right)\right\}$,

(5) $\left.\partial_{1}^{c}=\left\{\beth_{\partial_{1}},\right\urcorner_{\partial_{1}}, \beth_{\partial_{1}}\right\}$.

Definition 8. $[39,45,54]$ Let $\left.\partial_{1}=\left\{\beth_{\partial_{1}},\right\rceil_{\partial_{1}}, \beth_{\partial_{1}}\right\}$ and $\left.\partial_{2}=\left\{\beth_{\partial_{2}},\right\rceil_{\partial_{2}}, \beth_{\partial_{2}}\right\} \in \operatorname{SVNN}(\aleph)$ with $\ell>0$. than,

(1) $\left.\left.\left.\left.\partial_{1} \otimes \partial_{2}=\left\{\beth_{\partial_{1}} \beth_{\partial_{2}},\right\rceil_{\partial_{1}}+\right\rceil_{\partial_{2}}-\right\rceil_{\partial_{1}} \cdot\right\rceil_{\partial_{2}}, \beth_{\partial_{1}}+\beth_{\partial_{2}}-\beth_{\partial_{1}} \cdot \beth_{\partial_{2}}\right\}$;

(2) $\left.\left.\partial_{1} \boxplus \partial_{2}=\left\{\beth_{\partial_{1}}+\beth_{\partial_{2}}-\beth_{\partial_{1}} \beth_{\partial_{2}},\right\urcorner_{\partial_{1}}\right\urcorner_{\partial_{2}}, \beth_{\partial_{1}} \beth_{\partial_{2}}\right\}$;

(3) $\left(\partial_{1}\right)^{\ell}=\left\{\left(\beth_{\partial_{1}}\right)^{\ell}, 1-\left(1-\nearrow_{\partial_{1}}\right)^{\ell}, 1-\left(1-\beth_{\partial_{1}}\right)^{\ell}\right\}$;

(4) $\left.\ell \cdot \partial_{1}=\left\{1-\left(1-\beth_{\partial_{1}}\right)^{\ell},(\urcorner_{\partial_{1}}\right)^{\ell},\left(\beth_{\partial_{1}}\right)^{\ell}\right\}$;

(5) $\ell^{\partial_{1}}=\left\{\begin{array}{clc}\left(\ell^{\left.\left.1-\beth_{\partial_{1}}, 1-\ell\right\urcorner^{\partial_{1}}, 1-\ell^{\beth_{\partial_{1}}}\right)}\right. & \text { if } & \ell \in(0,1) \\ \left(\left(\frac{1}{\ell}\right)^{1-\beth_{\partial_{1}}}, 1-\left(\frac{1}{\ell}\right)^{\urcorner_{\partial_{1}}}, 1-\left(\frac{1}{\ell}\right)^{\beth_{\partial_{1}}}\right) & \text { if } & \ell \geq 1\end{array}\right.$.

Definition 9. Let $\left.\partial_{g}=\left\{\beth_{\partial_{g}},\right\urcorner_{\partial_{g}}, \beth_{\partial_{g}}\right\} \in \operatorname{SVNN}(\aleph)(g=1,2,3, \ldots, n)$. Then, the Algebraic averaging aggregation operator for SVNN ( $)$ is denoted by SVNWA and defined as follows,

$$
\begin{aligned}
\operatorname{SVNWA}\left(\partial_{1}, \partial_{2}, \partial_{3}, \ldots, \partial_{n}\right) & =\sum_{g=1}^{n} \ell_{g} \partial_{g}, \\
& \left.=\left\{1-\Pi_{g=1}^{n}\left(1-\beth_{\partial_{g}}\right)^{\ell_{g}}, \Pi_{g=1}^{n}(\rceil_{\partial_{g}}\right)^{\ell_{g}}, \Pi_{g=1}^{n}\left(\beth_{\partial_{g}}\right)^{\ell_{g}}\right\}
\end{aligned}
$$

where $\ell_{g}(g=1,2, \ldots, n)$ represents the weights of $\partial_{g}(g=1,2,3, \ldots, n)$ with $\ell_{g} \geq 0$ and $\sum_{g=1}^{n} \ell_{g}=1$.

Definition 10. Let $\left.\partial_{g}=\left\{\beth_{\partial_{g^{\prime}}},\right\urcorner_{\partial_{g^{\prime}}} \beth_{\partial_{g}}\right\} \in \operatorname{SVNN}(\aleph)(g=1,2,3, \ldots, n)$. Then, the Algebraic geometric aggregation operator for SVNN $(\aleph)$ is denoted by SVNWG and defined as follows,

$$
\begin{aligned}
\operatorname{SFWG}\left(\partial_{1}, \partial_{2}, \partial_{3}, \ldots, \partial_{n}\right) & =\prod_{g=1}^{n}\left(\partial_{g}\right)^{\ell_{g}}, \\
& \left.=\left\{\Pi_{g=1}^{n}\left(\beth_{\partial_{g}}\right)^{\ell_{g}}, 1-\Pi_{g=1}^{n}(1-\rceil_{\partial_{g}}\right)^{\ell_{g}}, 1-\Pi_{g=1}^{n}\left(1-\beth_{\partial_{g}}\right)^{\ell_{g}}\right\}
\end{aligned}
$$

where $\ell_{g}(g=1,2, \ldots, n)$ represents the weights of $\partial_{g}(g=1,2,3, \ldots, n)$ with $\ell_{g} \geq 0$ and $\sum_{g=1}^{n} \ell_{g}=1$.

\section{Novel Sine Trigonometric Operational Laws For SVNNs}

In this section, we propose the novel operational laws using sine trigonometric function under single-valued neutrosophic environments.

Definition 11. Let $\left.\partial=\left\{\beth_{\partial},\right\urcorner_{\partial}, \beth_{\partial}\right\} \in S V N N(\aleph)$. Then, sine trigonometric operational laws (STOLs) of SVNN $\partial$ is defined as follows,

$$
\sin (\partial)=\left\{\left(\begin{array}{c}
\left.\hbar, \sin \left(\frac{\pi}{2} \beth_{\partial}(\hbar)\right), 1-\sin \left(\frac{\pi}{2} 1-\right\rceil_{\partial}(\hbar)\right), \\
1-\sin \left(\frac{\pi}{2} 1-\beth_{\partial}(\hbar)\right)
\end{array}\right) \mid \hbar \in \aleph\right\}
$$

It is clearly seen that the $\sin (\partial)$ is also SVNS. As it is clear that, for each $\hbar \in \aleph$, the truth, indeterminacy, and falsity, $\left.\beth_{\partial}: \aleph \rightarrow \Theta,\right\rceil_{\partial}: \aleph \rightarrow \Theta$ and $\beth_{\partial}: \aleph \rightarrow \Theta$, respectively, of the element $\hbar$ to the SVNS $\partial$, where $\Theta=[0,1]$ be the unit interval. Furthermore, it is required that $\left.0 \leq \beth_{\partial}(\hbar)+\right\rceil_{\partial}(\hbar)+\beth_{\partial}(\hbar) \leq 3$, for each 
$\hbar \in \aleph$.

Furthermore, the truth membership grade

$$
\sin \left(\frac{\pi}{2} \beth_{\partial}\right): \aleph \rightarrow \Theta, \text { for each } \hbar \in \aleph \rightarrow \sin \left(\frac{\pi}{2} \beth_{\partial}(\hbar)\right) \in[0,1],
$$

indeterminacy membership grade

$$
\left.\left.1-\sin \left(\frac{\pi}{2} 1-\right\urcorner_{\partial}\right): \aleph \rightarrow \Theta, \text { for each } \hbar \in \aleph \rightarrow 1-\sin \left(\frac{\pi}{2} 1-\right\rceil_{\partial}(\hbar)\right) \in[0,1],
$$

and falsity membership grade

$$
1-\sin \left(\frac{\pi}{2} 1-\Xi_{\partial}\right): \aleph \rightarrow \Theta, \text { for each } \hbar \in \aleph \rightarrow 1-\sin \left(\frac{\pi}{2} 1-\Xi_{\partial}(\hbar)\right) \in[0,1] .
$$

Therefore,

$$
\sin (\partial)=\left\{\left(\begin{array}{c}
\left.\hbar, \sin \left(\frac{\pi}{2} \beth_{\partial}(\hbar)\right), 1-\sin \left(\frac{\pi}{2} 1-\right\rceil_{\partial}(\hbar)\right), \\
1-\sin \left(\frac{\pi}{2} 1-\beth_{\partial}(\hbar)\right)
\end{array}\right) \mid \hbar \in \aleph\right\}
$$

is SVNS.

Definition 12. Let $\left.\partial=\left\{\beth_{\partial},\right\rceil_{\partial}, \beth_{\partial}\right\} \in S V N N(\aleph)$. If

$$
\sin (\partial)=\left\{\left(\begin{array}{c}
\hbar, \sin \left(\frac{\pi}{2} \beth_{\partial}(\hbar)\right), 1-\sin \left(\frac{\pi}{2} 1-\nearrow_{\partial}(\hbar)\right), \\
1-\sin \left(\frac{\pi}{2} 1-\beth_{\partial}(\hbar)\right)
\end{array}\right) \mid \hbar \in \aleph\right\}
$$

Then, the function $\sin (\partial)$ is called the sine trigonometric operator and the value of $\sin (\partial)$ is called the sine trigonometric SVNN (STSVNN).

Theorem 1. Let $\left.\partial=\left\{\beth_{\partial},\right\rceil_{\partial}, \beth_{\partial}\right\} \in S V N N(\aleph)$. Then, the value of the operator $\sin (\partial)$ is $S V N N$.

Proof. As $\left.\partial=\left\{\beth_{\partial},\right\rceil_{\partial}, \beth_{\partial}\right\} \in S V N N(\aleph)$, that is, $\left.0 \leq \beth_{\partial} \leq 1,0 \leq\right\rceil_{\partial} \leq 1$ and $0 \leq \beth_{\partial} \leq 1$. Furthermore, $\beth_{\partial}(\hbar)+\nearrow_{\partial}(\hbar)+\beth_{\partial}(\hbar) \leq 3$, for each $\hbar \in \aleph$. To show $\sin (\partial)$ is SVNN, for this we have following two conditions.

(1) $\left.\sin \left(\frac{\pi}{2} \beth_{\partial}\right), 1-\sin \left(\frac{\pi}{2} 1-\right\rceil_{\partial}\right)$ and $1-\sin \left(\frac{\pi}{2} 1-\beth_{\partial}\right) \in[0,1]$

(2) $\left.\sin \left(\frac{\pi}{2} \beth_{\partial}\right)+1-\sin \left(\frac{\pi}{2} 1-\right\rceil_{\partial}\right)+1-\sin \left(\frac{\pi}{2} 1-\beth_{\partial}\right) \leq 3$.

As $0 \leq \beth_{\partial} \leq 1$ this implies that $0 \leq \frac{\pi}{2} \beth_{\partial} \leq \frac{\pi}{2}$. Also we know that "sin" is the increasing function in first quadrant, so we have $0 \leq \sin \left(\frac{\pi}{2} \beth_{\partial}\right) \leq 1$.

As $0 \leq 7_{\partial} \leq 1$ this implies that $\left.0 \leq \frac{\pi}{2} 1-\right\rceil_{\partial} \leq \frac{\pi}{2}$, which implies that $0 \leq \sin \left(\frac{\pi}{2} 1-7_{\partial}\right) \leq 1$. Thus, we get $\left.0 \leq 1-\sin \left(\frac{\pi}{2} 1-\right\rceil_{\partial}\right) \leq 1$. Similarly, we obtain $0 \leq 1-\sin \left(\frac{\pi}{2} 1-J_{\partial}\right) \leq 1$.

Therefore part (1) hold.

As $\left.\partial \in \operatorname{SVNN}(\aleph) \Rightarrow 0 \leq \beth_{\partial},\right\rceil_{\partial}, \beth_{\partial} \leq 1$ and $\left.\beth_{\partial}(\hbar)+\right\rceil_{\partial}(\hbar)+\beth_{\partial}(\hbar) \leq 3$, for each $\hbar \in \aleph$. Then (1) implies that $\left.0 \leq \sin \left(\frac{\pi}{2} \beth_{\partial}\right), 1-\sin \left(\frac{\pi}{2} 1-\right\rceil_{\partial}\right), 1-\sin \left(\frac{\pi}{2} 1-\beth_{\partial}\right) \leq 1$ and by Definition 11 , we have $\left.0 \leq \sin \left(\frac{\pi}{2} \beth_{\partial}\right)+1-\sin \left(\frac{\pi}{2} 1-\right\rceil_{\partial}\right)+1-\sin \left(\frac{\pi}{2} 1-\beth_{\partial}\right) \leq 3$.

Therefore, $\sin (\partial)$ is SVNN.

Definition 13. Let $\sin \left(\partial_{1}\right)=\left\{\left(\begin{array}{c}\sin \left(\frac{\pi}{2} \beth_{\partial_{1}}\right), \\ 1-\sin \left(\frac{\pi}{2} 1-\beth_{\partial_{1}}\right), \\ 1-\sin \left(\frac{\pi}{2} 1-\beth_{\partial_{1}}\right)\end{array}\right)\right\} \quad$ and $\quad \sin \left(\partial_{2}\right)=$ $\left\{\left(\begin{array}{c}\sin \left(\frac{\pi}{2} \beth_{\partial_{2}}\right), \\ \left.1-\sin \left(\frac{\pi}{2} 1-\right\rceil_{\partial_{2}}\right), \\ 1-\sin \left(\frac{\pi}{2} 1-\beth_{\partial_{2}}\right)\end{array}\right)\right\}$ be two STSVNNs. Then the operational laws are as follows 
(1) $\sin \left(\partial_{1}\right) \boxplus \sin \left(\partial_{2}\right)=\left(\begin{array}{c}1-\left(1-\sin \left(\frac{\pi}{2} \beth_{\partial_{1}}\right)\right)\left(1-\sin \left(\frac{\pi}{2} \beth_{\partial_{2}}\right)\right), \\ \left(1-\sin \left(\frac{\pi}{2} 1-\beth_{\partial_{1}}\right)\right)\left(1-\sin \left(\frac{\pi}{2} 1-\beth_{\partial_{2}}\right)\right), \\ \left(1-\sin \left(\frac{\pi}{2} 1-\beth_{\partial_{1}}\right)\right)\left(1-\sin \left(\frac{\pi}{2} 1-\beth_{\partial_{2}}\right)\right)\end{array}\right)$,

(2) $\psi \cdot \sin \left(\partial_{1}\right)=\left(\begin{array}{c}\left.1-\left(1-\sin \left(\frac{\pi}{2} \beth_{\partial_{1}}\right)\right)^{\psi},\left(1-\sin \left(\frac{\pi}{2} 1-\right\urcorner_{\partial_{1}}\right)\right)^{\psi}, \\ \left(1-\sin \left(\frac{\pi}{2} 1-\beth_{\partial_{1}}\right)\right)^{\psi}\end{array}\right)$,

(3) $\sin \left(\partial_{1}\right) \otimes \sin \left(\partial_{2}\right)=\left(\begin{array}{c}\sin \left(\frac{\pi}{2} \beth_{\partial_{1}}\right) \sin \left(\frac{\pi}{2} \beth_{\partial_{2}}\right), \\ \left.1-\left(\sin \left(\frac{\pi}{2} 1-\beth_{\partial_{1}}\right)\right)\left(\sin \left(\frac{\pi}{2} 1-\right\rceil_{\varkappa 2}\right)\right), \\ 1-\left(\sin \left(\frac{\pi}{2} 1-\beth_{\partial_{1}}\right)\right)\left(\sin \left(\frac{\pi}{2} 1-\beth_{\varkappa 2}\right)\right)\end{array}\right)$,

(4) $\left(\sin \left(\partial_{1}\right)\right)^{\psi}=\left(\begin{array}{c}\left(\sin \left(\frac{\pi}{2} \beth_{\partial_{1}}\right)\right)^{\psi}, \\ 1-\left(\sin \left(\frac{\pi}{2} 1-\beth_{\partial_{1}}\right)\right)^{\psi}, \\ 1-\left(\sin \left(\frac{\pi}{2} 1-\beth_{\partial_{1}}\right)\right)^{\prime}\end{array}\right)$.

To compare the STSVNNs, we have mentioned the following definitions.

Definition 14. [38] Let $\left.\partial=\left\{\beth_{\partial},\right\rceil_{\partial}, \beth_{\partial}\right\} \in S V N N(\aleph)$. Then, the score and accuracy of $\partial$ is denoted and defined as

(1) $\left.\overline{S C}(\partial)=\beth_{\partial}-\right\rceil_{\partial}-\beth_{\partial}$, and

(2) $\left.\underline{a c}(\partial)=\beth_{\partial}+\right\rceil_{\partial}+\beth_{\partial}$.

Definition 15. Let $\left.\partial_{1}=\left\{\beth_{\partial_{1}},\right\urcorner_{\partial_{1}}, \beth_{\partial_{1}}\right\}$ and $\left.\partial_{2}=\left\{\beth_{\partial_{2}},\right\rceil_{\partial_{2}}, \beth_{\partial_{2}}\right\} \in S V N N(\aleph)$. Then,

(1) If $\overline{s c}\left(\partial_{1}\right)<\overline{s c}\left(\partial_{2}\right)$ then $\partial_{1}<\partial_{2}$,

(2) If $\overline{s c}\left(\partial_{1}\right)>\overline{s c}\left(\partial_{2}\right)$ then $\partial_{1}>\partial_{2}$,

(3) If $\overline{s c}\left(\partial_{1}\right)=\overline{s c}\left(\partial_{2}\right)$ then

(a) $\underline{a c}\left(\partial_{1}\right)<\underline{a c}\left(\partial_{2}\right)$ then $\partial_{1}<\partial_{2}$,

(b) $\underline{a c}\left(\partial_{1}\right)>\underline{a c}\left(\partial_{2}\right)$ then $\partial_{1}>\partial_{2}$,

(c) $\underline{a c}\left(\partial_{1}\right)=\underline{a c}\left(\partial_{2}\right)$ then $\partial_{1}=\partial_{2}$.

Next we discussed some basic properties of STSVNNs based on proposed STOLs.

Theorem 2. Let $\left.\partial_{1}=\left\{\beth_{\partial_{1}},\right\urcorner_{\partial_{1}}, \beth_{\partial_{1}}\right\}$ and $\left.\partial_{2}=\left\{\beth_{\partial_{2}},\right\urcorner_{\partial_{2}}, \beth_{\partial_{2}}\right\} \in S V N N(\aleph)$. Then,

(1) $\sin \left(\partial_{1}\right) \boxplus \sin \left(\partial_{2}\right)=\sin \left(\partial_{2}\right) \boxplus \sin \left(\partial_{1}\right)$,

(2) $\sin \left(\partial_{1}\right) \otimes \sin \left(\partial_{2}\right)=\sin \left(\partial_{2}\right) \otimes \sin \left(\partial_{1}\right)$.

Proof. Straightforward from the Definition 12, so we omit the proofs of them.

Theorem 3. Let $\left.\partial_{g}=\left\{\beth_{\partial_{g}},\right\urcorner_{\partial_{g}}, \beth_{\partial_{g}}\right\} \in \operatorname{SVNN}(\aleph)(g=1,2,3)$. Then,

(1) $\left(\sin \left(\partial_{1}\right) \boxplus \sin \left(\partial_{2}\right)\right) \boxplus \sin \left(\partial_{3}\right)=\sin \left(\partial_{1}\right) \boxplus\left(\sin \left(\partial_{2}\right) \boxplus \sin \left(\partial_{3}\right)\right)$ ，

(2) $\left(\sin \left(\partial_{1}\right) \otimes \sin \left(\partial_{2}\right)\right) \otimes \sin \left(\partial_{3}\right)=\sin \left(\partial_{1}\right) \otimes\left(\sin \left(\partial_{2}\right) \otimes \sin \left(\partial_{3}\right)\right)$.

Proof. Straightforward from the Definition 12, so we omit the proofs of them.

Theorem 4. Let $\left.\partial_{g}=\left\{\beth_{\partial_{g^{\prime}}},\right\urcorner_{\partial_{g}}, \beth_{\partial_{g}}\right\} \in \operatorname{SVNN}(\aleph)(g=1,2)$ and $\psi, \psi_{1}, \psi_{2}>0$. Then,

(1) $\psi\left(\sin \left(\partial_{1}\right) \boxplus \sin \left(\partial_{2}\right)\right)=\psi \sin \left(\partial_{1}\right) \boxplus \psi \sin \left(\partial_{2}\right)$ ，

(2) $\left(\sin \left(\partial_{1}\right) \otimes \sin \left(\partial_{2}\right)\right)^{\psi}=\left(\sin \left(\partial_{1}\right)\right)^{\psi} \otimes\left(\sin \left(\partial_{2}\right)\right)^{\psi}$,

(3) $\psi_{1} \sin \left(\partial_{1}\right) \boxplus \psi_{2} \sin \left(\partial_{1}\right)=\left(\psi_{1}+\psi_{2}\right) \sin \left(\partial_{1}\right)$ ，

(4) $\left(\sin \left(\partial_{1}\right)\right)^{\psi_{1}} \nabla\left(\sin \left(\partial_{1}\right)\right)^{\psi_{2}}=\left(\sin \left(\partial_{1}\right)\right)^{\psi_{1}+\psi_{2}}$,

(5) $\left(\left(\sin \left(\partial_{1}\right)\right)^{\psi_{1}}\right)^{\psi_{2}}=\left(\sin \left(\partial_{1}\right)\right)^{\psi_{1} \cdot \psi_{2}}$. 
Proof. Let $\left.\partial_{g}=\left\{\beth_{\partial_{g^{\prime}}},\right\urcorner_{\partial_{g^{\prime}}} \beth_{\partial_{g}}\right\} \in S V N N(\aleph)(g=1,2)$ and $\psi, \psi_{1}, \psi_{2}>0$. Then, by the Definition 12 , we have $\sin \left(\partial_{1}\right)=\left\{\left(\begin{array}{c}\sin \left(\frac{\pi}{2} \beth_{\partial_{1}}\right), \\ \left.1-\sin \left(\frac{\pi}{2} 1-\right\rceil_{\partial_{1}}\right), \\ 1-\sin \left(\frac{\pi}{2} 1-\beth_{\partial_{1}}\right)\end{array}\right)\right\}$ and $\sin \left(\partial_{2}\right)=\left\{\left(\begin{array}{c}\sin \left(\frac{\pi}{2} \beth_{\partial_{2}}\right), \\ 1-\sin \left(\frac{\pi}{2} 1-\beth_{\partial_{2}}\right), \\ 1-\sin \left(\frac{\pi}{2} 1-\beth_{\partial_{2}}\right)\end{array}\right)\right\}$ be two STSVNNs. Therefore, using the STOLs for SVNNs, we obtain

$$
\sin \left(\partial_{1}\right) \boxplus \sin \left(\partial_{2}\right)=\left(\begin{array}{c}
1-\left(1-\sin \left(\frac{\pi}{2} \beth_{\partial_{1}}\right)\right)\left(1-\sin \left(\frac{\pi}{2} \beth_{\partial_{2}}\right)\right) \\
\left(1-\sin \left(\frac{\pi}{2} 1-\beth_{\partial_{1}}\right)\right)\left(1-\sin \left(\frac{\pi}{2} 1-\beth_{\partial_{2}}\right)\right) \\
\left(1-\sin \left(\frac{\pi}{2} 1-\beth_{\partial_{1}}\right)\right)\left(1-\sin \left(\frac{\pi}{2} 1-\beth_{\partial_{2}}\right)\right)
\end{array}\right) .
$$

(1) For any $\psi>0$, we have

$$
\begin{aligned}
& \psi\left(\sin \left(\partial_{1}\right) \boxplus \sin \left(\partial_{2}\right)\right) \\
= & \left(\begin{array}{c}
1-\left(1-\sin \left(\frac{\pi}{2} \beth_{\partial_{1}}\right)\right)^{\psi}\left(1-\sin \left(\frac{\pi}{2} \beth_{\partial_{2}}\right)\right)^{\psi}, \\
\left(\left(1-\sin \left(\frac{\pi}{2} 1-\beth_{\partial_{1}}\right)\right)\left(1-\sin ^{2}\left(\frac{\pi}{2} 1-\beth_{\partial_{2}}\right)\right)\right)^{\psi}, \\
\left(\left(1-\sin \left(\frac{\pi}{2} 1-\beth_{\partial_{1}}\right)\right)\left(1-\sin \left(\frac{\pi}{2} 1-\beth_{\partial_{2}}\right)\right)^{\psi}\right.
\end{array}\right) \\
= & \left(\begin{array}{c}
\left.1-\left(1-\sin \left(\frac{\pi}{2} \beth_{\partial_{1}}\right)\right)^{\psi},\left(1-\sin \left(\frac{\pi}{2} 1-\right\rceil_{\partial_{1}}\right)\right)^{\psi}, \\
\left(1-\sin \left(\frac{\pi}{2} 1-\beth_{\partial_{1}}\right)\right)^{\psi}
\end{array}\right) \\
& \boxplus\left(\begin{array}{c}
\left.1-\left(1-\sin \left(\frac{\pi}{2} \beth_{\partial_{2}}\right)\right)^{\psi},\left(1-\sin \left(\frac{\pi}{2} 1-\right\urcorner_{\partial_{2}}\right)\right)^{\psi}, \\
\left(1-\sin \left(\frac{\pi}{2} 1-\beth_{\partial_{2}}\right)\right)^{\psi}
\end{array}\right) \\
= & \psi \sin \left(\partial_{1}\right) \boxplus \psi \sin \left(\partial_{2}\right) .
\end{aligned}
$$

Proved.

(2) Proof is similarly as (1).

(3) For any $\psi_{1}, \psi_{2}>0$, we have

$$
\psi_{1} \sin \left(\partial_{1}\right)=\left(\begin{array}{c}
\left.1-\left(1-\sin \left(\frac{\pi}{2} \beth_{\partial_{1}}\right)\right)^{\psi_{1}},\left(1-\sin \left(\frac{\pi}{2} 1-\right\urcorner_{\partial_{1}}\right)\right)^{\psi_{1}}, \\
\left(1-\sin \left(\frac{\pi}{2} 1-\beth_{\partial_{1}}\right)\right)^{\psi_{1}}
\end{array}\right)
$$

and

$$
\psi_{2} \sin \left(\partial_{1}\right)=\left(\begin{array}{c}
\left.1-\left(1-\sin \left(\frac{\pi}{2} \beth_{\partial_{1}}\right)\right)^{\psi_{2}},\left(1-\sin \left(\frac{\pi}{2} 1-\right\rceil_{\partial_{1}}\right)\right)^{\psi_{2}}, \\
\left(1-\sin \left(\frac{\pi}{2} 1-\beth_{\partial_{1}}\right)\right)^{\psi_{2}}
\end{array}\right) .
$$

Thus, by STOLs for SVNNs, we get

$$
\begin{aligned}
& \psi_{1} \sin \left(\partial_{1}\right) \boxplus \psi_{2} \sin \left(\partial_{1}\right) \\
= & \left(\begin{array}{c}
\left.1-\left(1-\sin \left(\frac{\pi}{2} \beth_{\partial_{1}}\right)\right)^{\psi_{1}},\left(1-\sin \left(\frac{\pi}{2} 1-\right\urcorner_{\partial_{1}}\right)\right)^{\psi_{1}}, \\
\left(1-\sin \left(\frac{\pi}{2} 1-\beth_{\partial_{1}}\right)\right)^{\psi_{1}}
\end{array}\right) \\
& \boxplus\left(\begin{array}{c}
\left.1-\left(1-\sin \left(\frac{\pi}{2} \beth_{\partial_{1}}\right)\right)^{\psi_{2}},\left(1-\sin \left(\frac{\pi}{2} 1-\right\rceil_{\partial_{1}}\right)\right)^{\psi_{2}}, \\
\left(1-\sin \left(\frac{\pi}{2} 1-\beth_{\partial_{1}}\right)\right)^{\psi_{2}}
\end{array}\right) \\
= & \left(\begin{array}{c}
\left.1-\left(1-\sin \left(\frac{\pi}{2} \beth_{\partial_{1}}\right)\right)^{\psi_{1}+\psi_{2}},\left(1-\sin \left(\frac{\pi}{2} 1-\right\rceil_{\partial_{1}}\right)\right)^{\psi_{1}+\psi_{2}}, \\
\left(1-\sin \left(\frac{\pi}{2} 1-\beth_{\partial_{1}}\right)\right)^{\psi_{1}+\psi_{2}}
\end{array}\right) \\
= & \left(\psi_{1}+\psi_{2}\right) \sin \left(\partial_{1}\right)
\end{aligned}
$$

Proved.

$(4,5)$ Proof is similarly as (3). 
Theorem 5. Let $\left.\partial_{g}=\left\{\beth_{\partial_{g^{\prime}}}\right\rceil_{\partial_{g^{\prime}}} \beth_{\partial_{g}}\right\} \in \operatorname{SVNN}(\aleph)(g=1,2)$ such that $\left.\left.\beth_{\partial_{1}} \geq \beth_{\partial_{2^{\prime}}},\right\rceil_{\partial_{1}} \leq\right\rceil_{\partial_{2}}$ and $\beth_{\partial_{1}} \leq \beth_{\partial_{2}}$. Then

$$
\sin \left(\partial_{1}\right) \geq \sin \left(\partial_{2}\right)
$$

Proof. For $\left.\partial_{g}=\left\{\beth_{\partial_{g}},\right\urcorner_{\partial_{g^{\prime}}} \beth_{\partial_{g}}\right\} \in S V N N(\aleph)(g=1,2)$, we have $\beth_{\partial_{1}} \geq \beth_{\partial_{2}}$. As "sin" is an increasing function in $\left[0, \frac{\pi}{2}\right]$, thus we have $\sin \left(\frac{\pi}{2} \beth_{\partial_{1}}\right) \geq \sin \left(\frac{\pi}{2} \beth_{\partial_{2}}\right)$. Similarly, we have $\left.\rceil_{\partial_{1}} \leq\right\rceil_{\partial_{2}}$, which implies that $\left.1-\urcorner_{\partial_{1}} \geq 1-\right\urcorner_{\partial_{2}}$. Thus, $\left.\left.\sin \left(\frac{\pi}{2} 1-\right\urcorner_{\partial_{1}}\right) \geq \sin \left(\frac{\pi}{2} 1-\right\urcorner_{\partial_{1}}\right)$, which further implies that

$$
\left.\left.1-\sin \left(\frac{\pi}{2} 1-\right\urcorner_{\partial_{1}}\right) \leq 1-\sin \left(\frac{\pi}{2} 1-\right\urcorner_{\partial_{2}}\right)
$$

and similarly we get

$$
1-\sin \left(\frac{\pi}{2} 1-\beth_{\partial_{1}}\right) \leq 1-\sin \left(\frac{\pi}{2} 1-\beth_{\partial_{2}}\right)
$$

Therefore, we get

$$
\left\{\left(\begin{array}{c}
\sin \left(\frac{\pi}{2} \beth_{\partial_{1}}\right), \\
1-\sin \left(\frac{\pi}{2} 1-\beth_{\partial_{1}}\right), \\
1-\sin \left(\frac{\pi}{2} 1-\beth_{\partial_{1}}\right)
\end{array}\right)\right\} \geq\left\{\left(\begin{array}{c}
\sin \left(\frac{\pi}{2} \beth_{\partial_{2}}\right), \\
\left.1-\sin \left(\frac{\pi}{2} 1-\right\rceil_{\partial_{2}}\right), \\
1-\sin \left(\frac{\pi}{2} 1-\beth_{\partial_{2}}\right)
\end{array}\right)\right\} .
$$

Therefore, by Definition 12, we get $\sin \left(\partial_{1}\right) \geq \sin \left(\partial_{2}\right)$.

Proved.

\section{Novel Sine Trigonometric Aggregation Operators for SFNs}

In this section, we present some novel aggregation operators based on the proposed STOLs of SVNNs. we define the following weighted averaging and geometric aggregation operators (AOs).

\subsection{Sine Trigonometric Weighted Averaging AOs}

Definition 16. Let $\left.\partial_{g}=\left\{\beth_{\partial_{g}}(\hbar),\right\urcorner_{\partial_{g}}(\hbar), \beth_{\partial_{g}}(\hbar)\right\} \in S V N N(\aleph)(g=1,2,3, \ldots, n)$. Then, the sine trigonometric weighted averaging aggregation operator for SVNN $(\aleph)$ is denoted by ST - SVNWA and defined as follows:

$$
\begin{aligned}
S T-S V N W A\left(\partial_{1}, \partial_{2}, \ldots, \partial_{n}\right) & =\ell_{1} \sin \left(\partial_{1}\right) \boxplus \ell_{2} \sin \left(\partial_{2}\right) \boxplus \ldots \boxplus \ell_{n} \sin \left(\partial_{n}\right) \\
& =\sum_{g=1}^{n} \ell_{g} \sin \left(\partial_{g}\right),
\end{aligned}
$$

where $\ell_{g}(g=1,2, \ldots, n)$ represents the weights of $\partial_{g}(g=1,2,3, \ldots, n)$ with $\ell_{g} \geq 0$ and $\sum_{g=1}^{n} \ell_{g}=1$.

Theorem 6. Let $\left.\partial_{g}=\left\{\beth_{\partial_{g}}(\hbar),\right\rceil_{\partial_{g}}(\hbar), \beth_{\partial_{g}}(\hbar)\right\} \in \operatorname{SVNN}(\aleph)(g=1,2,3, \ldots, n)$ and the weight vector of $\partial_{g}(g=1,2,3, \ldots, n)$ be denoted by $\ell=\left(\ell_{1}, \ell_{2}, \ldots, \ell_{n}\right)^{T}$ subject to $\sum_{g=1}^{n} \ell_{g}=1$. The ST $-S V N W A$ operator is a mapping $G^{n} \longrightarrow$ G such that

$$
\begin{aligned}
S T-\operatorname{SVNWA}\left(\partial_{1}, \partial_{2}, \ldots, \partial_{n}\right)= & \sum_{g=1}^{n} \ell_{g} \sin \left(\partial_{g}\right) \\
= & \left(\begin{array}{c}
1-\prod_{g=1}^{n}\left(1-\sin \left(\frac{\pi}{2} \beth_{\partial_{g}}\right)\right)^{\ell_{g}}, \\
\left.\prod_{g=1}^{n}\left(1-\sin \left(\frac{\pi}{2} 1-\right\rceil_{\partial_{g}}\right)\right)^{\ell_{g}}, \\
\prod_{g=1}^{n}\left(1-\sin \left(\frac{\pi}{2} 1-\beth_{\partial_{g}}\right)\right)^{\ell_{g}}
\end{array}\right)
\end{aligned}
$$


Proof. We prove Theorem 6, by employing mathematical induction on $n$. As for each $g, \partial_{g}=$ $\left.\left\{\beth_{\partial_{g}}(\hbar),\right\rceil_{\partial_{g}}(\hbar), \beth_{\partial_{g}}(\hbar)\right\} \in S V N N(\aleph)$, which implies that $\left.\beth_{\partial_{g}},\right\rceil_{\partial_{g}}, \beth_{\partial_{g}} \in[0,1]$ and $\left.\beth_{\partial_{g}}+\right\rceil_{\partial_{g}}+$ $J_{\partial_{g}} \leq 3$. Then the following steps of the mathematical induction have been executed.

Step-1: For $n=2$, we get

$$
S T-S V N W A\left(\partial_{1}, \partial_{2}\right)=\ell_{1} \sin \left(\partial_{1}\right) \boxplus \ell_{2} \sin \left(\partial_{2}\right) .
$$

As by the Definition 12, we have $\sin \left(\partial_{1}\right)$ and $\sin \left(\partial_{2}\right)$ are SVNNs, and therefore $\ell_{1} \sin \left(\partial_{1}\right) \boxplus$ $\ell_{2} \sin \left(\partial_{2}\right)$ is also SVNN. Further, for $\partial_{1}$ and $\partial_{2}$, we have

$$
\begin{aligned}
& S T-\operatorname{SVNWA}\left(\partial_{1}, \partial_{2}\right) \\
= & \ell_{1} \sin \left(\partial_{1}\right) \boxplus \ell_{2} \sin \left(\partial_{2}\right) \\
= & \left(\begin{array}{c}
1-\left(1-\sin \left(\frac{\pi}{2} \beth_{\partial_{1}}\right)\right)^{\ell_{1}}, \\
\left(1-\sin \left(\frac{\pi}{2} 1-\beth_{\partial_{1}}\right)\right)^{\ell_{1}}, \\
\left(1-\sin \left(\frac{\pi}{2} 1-\beth_{\partial_{1}}\right)\right)^{\ell_{1}}
\end{array}\right) \boxplus\left(\begin{array}{c}
1-\left(1-\sin \left(\frac{\pi}{2} \beth_{\partial_{2}}\right)\right)^{\ell_{2}}, \\
\left(1-\sin \left(\frac{\pi}{2} 1-\beth_{\partial_{2}}\right)\right)^{\ell^{\prime}}, \\
\left(1-\sin \left(\frac{\pi}{2} 1-\beth_{\partial_{2}}\right)\right)^{\ell_{2}}
\end{array}\right) \\
= & \left(\begin{array}{c}
1-\prod_{g=1}^{2}\left(1-\sin \left(\frac{\pi}{2} \beth_{\partial_{g}}\right)\right)^{\ell_{g}}, \\
\left.\prod_{g=1}^{2}\left(1-\sin \left(\frac{\pi}{2} 1-\right\rceil_{\partial_{g}}\right)\right)^{\ell_{g}}, \\
\prod_{g=1}^{2}\left(1-\sin \left(\frac{\pi}{2} 1-\beth_{\partial_{g}}\right)\right)^{\ell_{g}}
\end{array}\right)
\end{aligned}
$$

Step-2: Suppose that Equation (1) is holds for $n=\kappa$, we have

$$
S T-\operatorname{SVNWA}\left(\partial_{1}, \partial_{2}, \ldots \partial_{\kappa}\right)=\left(\begin{array}{c}
1-\prod_{g=1}^{\kappa}\left(1-\sin \left(\frac{\pi}{2} \beth_{\partial_{g}}\right)\right)^{\ell_{g}}, \\
\left.\prod_{g=1}^{\kappa}\left(1-\sin \left(\frac{\pi}{2} 1-\right\rceil_{\partial_{g}}\right)\right)^{\ell_{g}}, \\
\prod_{g=1}^{\kappa}\left(1-\sin \left(\frac{\pi}{2} 1-\beth_{\partial_{g}}\right)\right)^{\ell_{g}}
\end{array}\right)
$$

Step-3: Now we have to prove that Equation (1) is holds for $n=\kappa+1$.

$$
\begin{aligned}
& \operatorname{ST}-\operatorname{SVNWA}\left(\partial_{1}, \partial_{2}, \ldots \partial_{\kappa+1}\right) \\
= & \sum_{g=1}^{\kappa} \ell_{g} \sin \left(\partial_{g}\right) \boxplus \ell_{\kappa+1} \sin \left(\partial_{\kappa+1}\right) \\
= & \left(\begin{array}{c}
1-\prod_{g=1}^{\kappa}\left(1-\sin \left(\frac{\pi}{2} \beth_{\partial_{g}}\right)\right)^{\ell_{g}}, \\
\left.\prod_{g=1}^{\kappa}\left(1-\sin \left(\frac{\pi}{2} 1-\right\rceil_{\partial_{g}}\right)\right)^{\ell_{g}}, \\
\prod_{g=1}^{\kappa}\left(1-\sin \left(\frac{\pi}{2} 1-\beth_{\partial_{g}}\right)\right)^{\ell_{g}}
\end{array}\right) \boxplus\left(\begin{array}{c}
1-\left(1-\sin \left(\frac{\pi}{2} \beth_{\partial_{\kappa+1}}\right)\right)^{\ell_{\kappa+1}}, \\
\left.\left(1-\sin \left(\frac{\pi}{2} 1-\right\rceil_{\partial_{\kappa+1}}\right)\right)^{\ell_{\kappa+1}}, \\
\left(1-\sin \left(\frac{\pi}{2} 1-\beth_{\partial_{\kappa+1}}\right)\right)^{\ell_{\kappa+1}}
\end{array}\right) \\
= & \left(\begin{array}{c}
1-\prod_{g=1}^{\kappa+1}\left(1-\sin \left(\frac{\pi}{2} \beth_{\partial_{g}}\right)\right)^{\ell_{g}}, \\
\left.\prod_{g=1}^{\kappa+1}\left(1-\sin \left(\frac{\pi}{2} 1-\right\rceil_{\partial_{g}}\right)\right)^{\ell_{g}}, \\
\prod_{g=1}^{\kappa+1}\left(1-\sin \left(\frac{\pi}{2} 1-\beth_{\partial_{g}}\right)\right)^{\ell_{g}}
\end{array}\right)
\end{aligned}
$$

that is, when $n=z+1$, Equation (1) also holds. 
Therefore, Equation (1) holds for any $n$. The proof is completed.

Example 1. Suppose $\partial_{1}=\{0.61,0.15,0.53\}, \partial_{2}=\{0.16,0.35,0.62\}, \partial_{3}=\{0.56,0.17,0.44\}$, and $\partial_{4}=$ $\{0.37,0.32,0.65\}$ are the single-valued neutrosophic numbers with $\ell=(0.256,0.248,0.245,0.251)^{T}$ is the weight vector.

First, we find the $l_{g}=\sin ^{2}\left(\frac{\pi}{2} \beth_{\partial_{g}}\right)$ we get

$$
\begin{array}{ll}
l_{1}=0.6693 & l_{2}=0.0618 \\
l_{3}=0.5936 & l_{4}=0.3012
\end{array}
$$

Thus, we have

$$
\begin{aligned}
\prod_{g=1}^{4}\left(1-\sin ^{2}\left(\frac{\pi}{2} \beth_{\partial_{g}}\right)\right)^{\ell_{g}} & =\left(1-l_{1}\right)^{0.256} \times\left(1-l_{2}\right)^{0.248} \times\left(1-l_{3}\right)^{0.245} \times\left(1-l_{4}\right)^{0.251} \\
& =0.7533 \times 0.9843 \times 0.8020 \times 0.9139 \\
& =0.5434
\end{aligned}
$$

Similarly, if $m_{g}=\sin ^{2}\left(\frac{\pi}{2} \sqrt{1-7_{\partial_{g}}^{2}}\right)$, we get

$$
\begin{array}{ll}
m_{1}=0.9996 & m_{2}=0.9901 \\
m_{3}=0.9994 & m_{4}=0.9931
\end{array}
$$

Thus, we have

$$
\begin{aligned}
\prod_{g=1}^{4}\left(\sqrt{\left.1-\sin ^{2}\left(\frac{\pi}{2} \sqrt{1-\tau_{\partial_{g}}^{2}}\right)\right)^{\ell_{g}}=}\right. & \left(\sqrt{1-m_{1}}\right)^{0.256} \times\left(\sqrt{1-m_{2}}\right)^{0.248} \\
& \times\left(\sqrt{1-m_{3}}\right)^{0.245} \times\left(\sqrt{1-m_{4}}\right)^{0.251} \\
= & 0.3673 \times 0.5642 \times 0.5343 \times 0.5355 \\
= & 0.0592
\end{aligned}
$$

Similarly, if $n_{g}=\sin ^{2}\left(\frac{\pi}{2} \sqrt{1-\beth_{\partial_{g}}^{2}}\right)$, we get

$$
\begin{array}{ll}
m_{1}=0.9440 & m_{2}=0.8898 \\
m_{3}=0.9745 & m_{4}=0.8644
\end{array}
$$

Thus, we have

$$
\begin{aligned}
\prod_{g=1}^{4}\left(\sqrt{\left.1-\sin ^{2}\left(\frac{\pi}{2} \sqrt{1-J_{\partial_{g}}^{2}}\right)\right)^{\ell_{g}}=}\right. & \left(\sqrt{1-n_{1}}\right)^{0.256} \times\left(\sqrt{1-n_{2}}\right)^{0.248} \\
& \times\left(\sqrt{1-n_{3}}\right)^{0.245} \times\left(\sqrt{1-n_{4}}\right)^{0.251} \\
= & 0.6914 \times 0.7607 \times 0.6379 \times 0.7782 \\
= & 0.2611
\end{aligned}
$$


Therefore,

$$
\begin{aligned}
S T-\operatorname{SVNWA}\left(\partial_{1}, \partial_{2}, \partial_{3}, \partial_{4}\right) & =\left(\begin{array}{c}
\sqrt{1-\prod_{g=1}^{4}\left(1-\sin ^{2}\left(\frac{\pi}{2} \beth_{\partial_{g}}\right)\right)^{\ell_{g}}}, \\
\prod_{g=1}^{4}\left(\sqrt{1-\sin ^{2}\left(\frac{\pi}{2} \sqrt{1-\rceil_{\partial_{g}}^{2}}\right)}\right)^{\ell_{g}}, \\
\prod_{g=1}^{4}\left(\sqrt{1-\sin ^{2}\left(\frac{\pi}{2} \sqrt{1-\beth_{\partial_{g}}^{2}}\right)}\right)^{\ell_{g}}
\end{array}\right) \\
& =(\sqrt{1-0.5434}, 0.0592,0.2611) \\
& =(0.6757,0.0592,0.2611)
\end{aligned}
$$

Next, we give the some properties of the proposed ST - SVNWA aggregation operator. As these aggregation operators are based on the sine trigonometric function, they preserve the idempotency, boundedness, monotonically, and symmetry.

Theorem 7. (Idempotency) Let $\left.\partial_{g}=\left\{\beth_{\partial_{g}}(\hbar),\right\urcorner_{\partial_{g}}(\hbar), \beth_{\partial_{g}}(\hbar)\right\} \in S V N N(\aleph)(g=1,2,3, \ldots, n)$ such that $\partial_{g}=\partial$. Then

$$
S T-\operatorname{SVNWA}\left(\partial_{1}, \partial_{2}, \ldots, \partial_{n}\right)=\sin (\partial) \text {. }
$$

Proof. As $\partial_{g}=\partial(g=1,2,3, \ldots, n)$. Then, by Theorem 6 , we get

$$
\begin{aligned}
S T-\operatorname{SVNWA}\left(\partial_{1}, \partial_{2}, \ldots, \partial_{n}\right)= & \left(\begin{array}{c}
1-\prod_{g=1}^{n}\left(1-\sin \left(\frac{\pi}{2} \beth_{\partial_{g}}\right)\right)^{\ell_{g}}, \\
\left.\prod_{g=1}^{n}\left(1-\sin \left(\frac{\pi}{2} 1-\right\rceil_{\partial_{g}}\right)\right)^{\ell_{g}}, \\
\prod_{g=1}^{n}\left(1-\sin \left(\frac{\pi}{2} 1-\beth_{\partial_{g}}\right)\right)^{\ell_{g}}
\end{array}\right) \\
= & \left(\begin{array}{c}
1-\prod_{g=1}^{n}\left(1-\sin \left(\frac{\pi}{2} \beth_{\partial}\right)\right)^{\ell_{g},} \\
\left.\prod_{g=1}^{n}\left(1-\sin \left(\frac{\pi}{2} 1-\right\rceil_{\partial}\right)\right)^{\ell_{g}}, \\
\prod_{g=1}^{n}\left(1-\sin \left(\frac{\pi}{2} 1-\beth_{\partial}\right)\right)^{\ell_{g}}
\end{array}\right) \\
= & \left(\begin{array}{c}
1-\left(1-\sin \left(\frac{\pi}{2} \beth_{\partial}\right)\right)^{\sum_{g=1}^{n} \ell_{g},} \\
\left.\left(1-\sin \left(\frac{\pi}{2} 1-\right\rceil_{\partial}\right)\right)^{\sum_{g=1}^{n} \ell_{g},} \\
\left(1-\sin \left(\frac{\pi}{2} 1-\beth_{\partial}\right)\right)^{\sum_{g=1}^{n} \ell_{g}}
\end{array}\right) \\
= & \left(\begin{array}{c}
\left.\sin \left(\frac{\pi}{2} \beth_{\partial}\right), 1-\sin \left(\frac{\pi}{2} 1-\right\rceil_{\partial}\right), \\
1-\sin \left(\frac{\pi}{2} 1-\beth_{\partial}\right)
\end{array}\right) \\
= & \sin (\partial)
\end{aligned}
$$

Proved.

Theorem 8. (Boundedness) Let $\left.\partial_{g}=\left\{\beth_{\partial_{g}}(\hbar),\right\rceil_{\partial_{g}}(\hbar), \beth_{\partial_{g}}(\hbar)\right\}, \partial_{g}^{-}=$ $\left\{\min \left(\beth_{\partial_{g}}(\hbar)\right), \max \left(\nearrow_{\partial_{g}}(\hbar)\right), \max \left(\beth_{\partial_{g}}(\hbar)\right)\right\}$ and $\partial_{g}^{+}=\left\{\max \left(\beth_{\partial_{g}}(\hbar)\right), \min \left(\nearrow_{\partial_{g}}(\hbar)\right), \min \left(\beth_{\partial_{g}}(\hbar)\right)\right\}$ $\in \operatorname{SVNN}(\aleph)(g=1,2,3, \ldots, n)$. Then,

$$
\sin \left(\partial_{g}^{-}\right) \leq S T-S V N W A\left(\partial_{1}, \partial_{2}, \ldots, \partial_{n}\right) \leq \sin \left(\partial_{g}^{+}\right) .
$$


Symmetry 2020, 12, 298

13 of 27

Proof. As, for any $\left.g, \min _{g}\left(\beth_{\partial_{g}}\right) \leq \beth_{\partial_{g}} \leq \min _{g}\left(\beth_{\partial_{g}}\right), \min _{g}\left(\nearrow_{\partial_{g}}\right) \leq\right\rceil_{\partial_{g}} \leq \min _{g}\left(\nearrow_{\partial_{g}}\right)$ and $\min _{g}\left(\beth_{\partial_{g}}\right) \leq \beth_{\partial_{g}} \leq \min _{g}\left(\beth_{\partial_{g}}\right)$. This implies that $\partial_{g}^{-} \leq \partial_{g} \leq \partial_{g}^{+}$.

Suppose that $S T-\operatorname{SVNWA}\left(\partial_{1}, \partial_{2}, \ldots, \partial_{n}\right)=\sin \left(\partial_{g}\right)=\left\{\beth_{\partial_{g}}, \neg_{\partial_{g}}, \beth_{\partial_{g}}\right\}, \sin \left(\partial_{g}^{-}\right)=$ $\left\{\beth_{\partial_{g}}^{-}, \neg_{\partial_{g}}^{-}, \beth_{\partial_{g}}^{-}\right\}$and $\sin \left(\partial_{g}^{+}\right)=\left\{\beth_{\partial_{g}}^{+}, \neg_{\partial_{g}}^{+}, \beth_{\partial_{g}}^{+}\right\}$. Then, based on the monotonicity of sine function, we have

$$
\begin{aligned}
\beth_{\partial_{g}} & =1-\prod_{g=1}^{n}\left(1-\sin \left(\frac{\pi}{2} \beth_{\partial_{g}}\right)\right)^{\ell_{g}} \\
& \geq 1-\prod_{g=1}^{n}\left(1-\sin \left(\frac{\pi}{2} \min _{g}\left(\beth_{\partial_{g}}\right)\right)\right)^{\ell_{g}} \\
& =\sin \left(\frac{\pi}{2} \min _{g}\left(\beth_{\partial_{g}}\right)\right)=\beth_{\partial_{g}}^{-}
\end{aligned}
$$

and,

$$
\begin{aligned}
\rceil_{\partial_{g}} & \left.=\prod_{g=1}^{n}\left(1-\sin \left(\frac{\pi}{2} 1-\right\rceil_{\partial_{g}}\right)\right)^{\ell_{g}} \\
& \left.\geq \prod_{g=1}^{n}\left(1-\sin \left(\frac{\pi}{2} 1-\left(\min _{g}\right\rceil_{\partial_{g}}\right)\right)\right)^{\ell_{g}} \\
& \left.=1-\sin \left(\frac{\pi}{2} 1-\left(\min _{g}\right\rceil_{\partial_{g}}\right)\right)=\neg_{\partial_{g}}^{-}
\end{aligned}
$$

Similarly,

$$
\begin{aligned}
\beth_{\partial_{g}} & =\prod_{g=1}^{n}\left(1-\sin \left(\frac{\pi}{2} 1-\beth_{\partial_{g}}\right)\right)^{\ell_{g}} \\
& \geq \prod_{g=1}^{n}\left(1-\sin \left(\frac{\pi}{2} 1-\left(\min _{g} \beth_{\partial_{g}}\right)\right)\right)^{\ell_{g}} \\
& =1-\sin \left(\frac{\pi}{2} 1-\left(\min _{g} \beth_{\partial_{g}}\right)\right)=\beth_{\partial_{g}}^{-}
\end{aligned}
$$

Also, we have

$$
\begin{aligned}
\beth_{\partial_{g}} & =\sqrt{1-\prod_{g=1}^{n}\left(1-\sin ^{2}\left(\frac{\pi}{2} \beth_{\partial_{g}}\right)\right)^{\ell_{g}}} \\
& \leq \sqrt{1-\prod_{g=1}^{n}\left(1-\sin ^{2}\left(\frac{\pi}{2} \max _{g}\left(\beth_{\partial_{g}}\right)\right)\right)^{\ell_{g}}} \\
& =\sin \left(\frac{\pi}{2} \max _{g}\left(\beth_{\partial_{g}}\right)\right)=\beth_{\partial_{g}}^{+}
\end{aligned}
$$

and

$$
\begin{aligned}
\rceil_{\partial_{g}} & \left.=\prod_{g=1}^{n}\left(1-\sin \left(\frac{\pi}{2} 1-\right\rceil_{\partial_{g}}\right)\right)^{\ell_{g}} \\
& \left.\leq \prod_{g=1}^{n}\left(1-\sin \left(\frac{\pi}{2} \cdot 1-\left(\max _{g}\right\rceil_{\partial_{g}}\right)\right)\right)^{\ell_{g}} \\
& \left.=1-\sin \left(\frac{\pi}{2} \cdot 1-\left(\max _{g}\right\rceil_{\partial_{g}}\right)\right)=7_{\partial_{g}}^{+}
\end{aligned}
$$


Similarly,

$$
\begin{aligned}
\beth_{\partial_{g}} & =\prod_{g=1}^{n}\left(1-\sin \left(\frac{\pi}{2} 1-\beth_{\partial_{g}}\right)\right)^{\ell_{g}} \\
& \leq \prod_{g=1}^{n}\left(1-\sin \left(\frac{\pi}{2} 1-\left(\max _{g} \beth_{\partial_{g}}\right)\right)\right)^{\ell_{g}} \\
& =1-\sin \left(\frac{\pi}{2} 1-\left(\max _{g} \beth_{\partial_{g}}\right)\right)=\beth_{\partial_{g}^{+}}^{+}
\end{aligned}
$$

Based on the sore function, we get

$$
\begin{aligned}
\overline{s c}\left(\sin \left(\partial_{g}\right)\right) & \left.=\beth_{\partial_{g}}-\right\rceil_{\partial_{g}}-\beth_{\partial_{g}} \\
& \left.\leq \beth_{\partial_{g}}^{+}-\right\urcorner_{\partial_{g}}^{-}-\beth_{\partial_{g}}^{-}=\overline{s C}\left(\sin \left(\partial_{g}^{+}\right)\right)
\end{aligned}
$$

and

$$
\begin{aligned}
\overline{s c}\left(\sin \left(\partial_{g}\right)\right) & \left.=\beth_{\partial_{g}}-\right\urcorner_{\partial_{g}}-\beth_{\partial_{g}} \\
& \left.\geq \beth_{\partial_{g}}^{-}-\right\rceil_{\partial_{g}}^{+}-\beth_{\partial_{g}}^{+}=\overline{s c}\left(\sin \left(\partial_{g}^{-}\right)\right)
\end{aligned}
$$

Therefore, $\overline{s c}\left(\sin \left(\partial_{g}^{-}\right)\right) \leq \overline{s c}\left(\sin \left(\partial_{g}\right)\right) \leq \overline{s c}\left(\sin \left(\partial_{g}^{+}\right)\right)$. Now, we discuss the three cases:

(Case-1): If $\overline{s c}\left(\sin \left(\partial_{g}^{-}\right)\right)<\overline{s c}\left(\sin \left(\partial_{g}\right)\right)<\overline{s c}\left(\sin \left(\partial_{g}^{+}\right)\right)$, then the result holds.

(Case-2): If $\overline{s c}\left(\sin \left(\partial_{g}^{+}\right)\right)=\overline{s c}\left(\sin \left(\partial_{g}\right)\right)$ then $\left.\left.\beth_{\partial_{g}}^{+}-\right\rceil_{\partial_{g}}^{+}-\beth_{\partial_{g}}^{+}=\beth_{\partial_{g}}-\right\rceil_{\partial_{g}}-\beth_{\partial_{g}}$, which implies that $\left.\left.\beth_{\partial_{g}}^{+}=\beth_{\partial_{g}},\right\urcorner_{\partial_{g}}^{+}=\right\urcorner_{\partial_{g}}$ and $\beth_{\partial_{g}}^{+}=\beth_{\partial_{g}}$, and therefore $\underline{a c}\left(\sin \left(\partial_{g}\right)\right)=\underline{a c}\left(\sin \left(\partial_{g}^{+}\right)\right)$.

(Case-3): If $\overline{s c}\left(\sin \left(\partial_{g}\right)\right)=\overline{s c}\left(\sin \left(\partial_{g}^{-}\right)\right)$then $\left.\left.\beth_{\partial_{g}}-\right\urcorner_{\partial_{g}}-\beth_{\partial_{g}}=\beth_{\partial_{g}}^{-}-\right\urcorner_{\partial_{g}}^{-}-\beth_{\partial_{g}}^{-}$which implies that $\left.\left.\beth_{\partial_{g}}=\beth_{\partial_{g^{\prime}}}^{-},\right\urcorner_{\partial_{g}}=\right\urcorner_{\partial_{g}}^{-}$and $\beth_{\partial_{g}}=\beth_{\partial_{g^{\prime}}}^{-}$and therefore $\underline{a c}\left(\sin \left(\partial_{g}\right)\right)=\underline{a c}\left(\sin \left(\partial_{g}^{-}\right)\right)$. Therefore, we finally obtain

$$
\sin \left(\partial_{g}^{-}\right) \leq S T-S V N W A\left(\partial_{1}, \partial_{2}, \ldots, \partial_{n}\right) \leq \sin \left(\partial_{g}^{+}\right)
$$

Proved.

Theorem 9. (Monotonically) Let $\left.\partial_{g}=\left\{\beth_{\partial_{g}}(\hbar),\right\rceil_{\partial_{g}}(\hbar), \beth_{\partial_{g}}(\hbar)\right\}, \partial_{g}^{*}=\left\{\beth_{\partial_{g}}^{*}(\hbar), \neg_{\partial_{g}}^{*}(\hbar), \beth_{\partial_{g}}^{*}(\hbar)\right\} \in$ $\operatorname{SVNN}(\aleph)(g=1,2,3, \ldots, n)$. If $\left.\left.\beth_{\partial_{g}} \leq \beth_{\partial_{g^{\prime}}^{*}}^{*}\right\rceil_{\partial_{g}} \leq\right\rceil_{\partial_{g}}^{*}$ and $\beth_{\partial_{g}} \leq \beth_{\partial_{g^{\prime}}}^{*}$, then

$$
S T-S V N W A\left(\partial_{1}, \partial_{2}, \ldots, \partial_{n}\right) \leq S T-S V N W A\left(\partial_{1}^{*}, \partial_{2}^{*}, \ldots, \partial_{n}^{*}\right) \text {. }
$$

Proof. Follows from Theorem 8, so we omit here.

Theorem 10. (Symmetric) Let $\left.\partial_{g}=\left\{\beth_{\partial_{g}}(\hbar),\right\rceil_{\partial_{g}}(\hbar), \beth_{\partial_{g}}(\hbar)\right\}, \partial_{g}^{*}=\left\{\beth_{\partial_{g}^{*}}^{*}(\hbar), \neg_{\partial_{g}^{*}}^{*}(\hbar), \beth_{\partial_{g}^{*}}^{*}(\hbar)\right\} \in$ $\operatorname{SVNN}(\aleph)(g=1,2,3, \ldots, n)$. Then,

$$
S T-S V N W A\left(\partial_{1}, \partial_{2}, \ldots, \partial_{n}\right)=S T-S V N W A\left(\partial_{1}^{*}, \partial_{2}^{*}, \ldots, \partial_{n}^{*}\right),
$$

whenever $\partial_{g}^{*}(g=1,2,3, \ldots, n)$ is any version of $\partial_{g}(g=1,2,3, \ldots, n)$.

Proof. Follows from Theorem 8, so we omit here. 
Definition 17. Let $\left.\partial_{g}=\left\{\beth_{\partial_{g}}(\hbar),\right\rceil_{\partial_{g}}(\hbar), \beth_{\partial_{g}}(\hbar)\right\} \in \operatorname{SVNN}(\aleph)(g=1,2,3, \ldots, n)$. Then, the sine trigonometric ordered weighted averaging aggregation operator for SVNN ( $)$ is denoted by ST - SVNOWA and defined as follows,

$$
\begin{aligned}
\operatorname{ST}-\operatorname{SVNOWA}\left(\partial_{1}, \partial_{2}, \ldots, \partial_{n}\right) & =\ell_{1} \sin \left(\partial_{v(1)}\right) \boxplus \ell_{2} \sin \left(\partial_{v(2)}\right) \boxplus \ldots \boxplus \ell_{n} \sin \left(\partial_{v(n)}\right) \\
& =\sum_{g=1}^{n} \ell_{g} \sin \left(\partial_{v(g)}\right)
\end{aligned}
$$

where $v(g)$ is denoted for ordered and $(v(1), v(2), v(3), \ldots, v(n))$ is a permutation of $(1,2,3, \ldots, n)$, subject to $\varepsilon_{v(g-1)} \geq \varepsilon_{v(g)}$ for all $g$. Also, $\ell_{g}(g=1,2, \ldots, n)$ represents the weights of $\partial_{g}(g=1,2,3, \ldots, n)$ with $\ell_{g} \geq 0$ and $\sum_{g=1}^{n} \ell_{g}=1$.

Theorem 11. Let $\left.\partial_{g}=\left\{\beth_{\partial_{g}}(\hbar),\right\urcorner_{\partial_{g}}(\hbar), \beth_{\partial_{g}}(\hbar)\right\} \in S V N N(\aleph)(g=1,2,3, \ldots, n)$ and the weight vector of $\partial_{g}(g=1,2,3, \ldots, n)$ be denoted by $\ell=\left(\ell_{1}, \ell_{2}, \ldots, \ell_{n}\right)^{T}$ subject to $\sum_{g=1}^{n} \ell_{g}=1$. The ST - SVNOWA operator is a mapping $G^{n} \longrightarrow$ Guch that

$$
\begin{aligned}
\operatorname{ST}-\operatorname{SVNOWA}\left(\partial_{1}, \partial_{2}, \ldots, \partial_{n}\right)= & \sum_{g=1}^{n} \ell_{g} \sin \left(\partial_{v(g)}\right) \\
& =\left(\begin{array}{c}
1-\prod_{g=1}^{n}\left(1-\sin \left(\frac{\pi}{2} \beth_{\partial_{v(g)}}\right)\right)^{\ell_{g}}, \\
\left.\prod_{g=1}^{n}\left(1-\sin \left(\frac{\pi}{2} \cdot 1-\right\rceil_{\partial_{v(g)}}\right)\right)^{\ell_{g}}, \\
\prod_{g=1}^{n}\left(1-\sin \left(\frac{\pi}{2} \cdot 1-\beth_{\partial_{v(g)}}\right)\right)^{\ell_{g}}
\end{array}\right)
\end{aligned}
$$

Proof. Follows from Theorem 6 similarly.

Theorem 12. (Idempotency) Let $\left.\partial_{g}=\left\{\beth_{\partial_{g}}(\hbar),\right\urcorner_{\partial_{g}}(\hbar), \beth_{\partial_{g}}(\hbar)\right\} \in \operatorname{SVNN}(\aleph)(g=1,2,3, \ldots, n)$ such that $\partial_{g}=\partial$. Then

$$
S T-\operatorname{SVNOWA}\left(\partial_{1}, \partial_{2}, \ldots, \partial_{n}\right)=\sin (\partial) \text {. }
$$

Theorem 13. (Boundedness) Let $\left.\partial_{g}=\left\{\beth_{\partial_{g}}(\hbar),\right\rceil_{\partial_{g}}(\hbar), \beth_{\partial_{g}}(\hbar)\right\}, \partial_{g}^{-}=$ $\left\{\min \left(\beth_{\partial_{g}}(\hbar)\right), \max \left(\nearrow_{\partial_{g}}(\hbar)\right), \max \left(\beth_{\partial_{g}}(\hbar)\right)\right\}$ and $\partial_{g}^{+}=\left\{\max \left(\beth_{\partial_{g}}(\hbar)\right), \min \left(\nearrow_{\partial_{g}}(\hbar)\right), \min \left(\beth_{\partial_{g}}(\hbar)\right)\right\}$ $\in \operatorname{SVNN}(\aleph)(g=1,2,3, \ldots, n)$. Then,

$$
\sin \left(\partial_{g}^{-}\right) \leq S T-S V N O W A\left(\partial_{1}, \partial_{2}, \ldots, \partial_{n}\right) \leq \sin \left(\partial_{g}^{+}\right) .
$$

Theorem 14. (Monotonically) Let $\left.\left.\partial_{g}=\left\{\beth_{\partial_{g}}(\hbar),\right\rceil_{\partial_{g}}(\hbar), \beth_{\partial_{g}}(\hbar)\right\}, \partial_{g}^{*}=\left\{\beth_{\partial_{g}}^{*}(\hbar),\right\rceil_{\partial_{g}}^{*}(\hbar), \beth_{\partial_{g}}^{*}(\hbar)\right\} \in$ $\operatorname{SVNN}(\aleph)(g=1,2,3, \ldots, n)$. If $\left.\left.\beth_{\partial_{g}} \leq \beth_{\partial_{g^{\prime}}}^{*}\right\urcorner_{\partial_{g}} \leq\right\rceil_{\partial_{g}}^{*}$ and $\beth_{\partial_{g}} \leq \beth_{\partial_{g^{\prime}}}^{*}$, then

$$
S T-\operatorname{SVNOWA}\left(\partial_{1}, \partial_{2}, \ldots, \partial_{n}\right) \leq S T-S V N O W A\left(\partial_{1}^{*}, \partial_{2}^{*}, \ldots, \partial_{n}^{*}\right) \text {. }
$$

Theorem 15. (Symmetric) Let $\left.\partial_{g}=\left\{\beth_{\partial_{g}}(\hbar),\right\rceil_{\partial_{g}}(\hbar), \beth_{\partial_{g}}(\hbar)\right\}, \partial_{g}^{*}=\left\{\beth_{\partial_{g}^{*}}^{*}(\hbar), \neg_{\partial_{g}^{*}}^{*}(\hbar), \beth_{\partial_{g}}^{*}(\hbar)\right\} \in$ $\operatorname{SVNN}(\aleph)(g=1,2,3, \ldots, n)$. Then,

$$
S T-\operatorname{SVNOWA}\left(\partial_{1}, \partial_{2}, \ldots, \partial_{n}\right)=S T-\operatorname{SVNOWA}\left(\partial_{1}^{*}, \partial_{2}^{*}, \ldots, \partial_{n}^{*}\right),
$$

whenever $\partial_{g}^{*}(g=1,2,3, \ldots, n)$ is any version of $\partial_{g}(g=1,2,3, \ldots, n)$. 
Proof of above theorems are follows form Theorems 7-10 similarly.

\subsection{Sine Trigonometric Weighted Geometric AOs}

Definition 18. Let $\left.\partial_{g}=\left\{\beth_{\partial_{g}}(\hbar),\right\urcorner_{\partial_{g}}(\hbar), \beth_{\partial_{g}}(\hbar)\right\} \in S V N N(\aleph)(g=1,2,3, \ldots, n)$. Then, the sine trigonometric weighted geometric aggregation operator for SVNN $(\aleph)$ is denoted by ST - SVNWG and defined as follows:

$$
\begin{aligned}
S T-S V N W G\left(\partial_{1}, \partial_{2}, \ldots, \partial_{n}\right) & =\left(\sin \left(\partial_{1}\right)\right)^{\ell_{1}} \otimes\left(\sin \left(\partial_{2}\right)\right)^{\ell_{2}} \otimes \ldots \otimes\left(\sin \left(\partial_{n}\right)\right)^{\ell_{n}} \\
& =\prod_{g=1}^{n}\left(\sin \left(\partial_{g}\right)\right)^{\ell_{g}}
\end{aligned}
$$

where $\ell_{g}(g=1,2, \ldots, n)$ represents the weights of $\partial_{g}(g=1,2,3, \ldots, n)$ with $\ell_{g} \geq 0$ and $\sum_{g=1}^{n} \ell_{g}=1$.

Theorem 16. Let $\left.\partial_{g}=\left\{\beth_{\partial_{g}}(\hbar),\right\urcorner_{\partial_{g}}(\hbar), \beth_{\partial_{g}}(\hbar)\right\} \in S V N N(\aleph)(g=1,2,3, \ldots, n)$ and the weight vector of $\partial_{g}(g=1,2,3, \ldots, n)$ be denoted by $\ell=\left(\ell_{1}, \ell_{2}, \ldots, \ell_{n}\right)^{T}$ subject to $\sum_{g=1}^{n} \ell_{g}=1$. The ST - SVNWG operator is a mapping $G^{n} \longrightarrow$ G such that

$$
\begin{aligned}
S T-\operatorname{SVNWG}\left(\partial_{1}, \partial_{2}, \ldots, \partial_{n}\right)= & \prod_{g=1}^{n}\left(\sin \left(\partial_{g}\right)\right)^{\ell_{g}} \\
& =\left(\begin{array}{c}
\prod_{g=1}^{n}\left(\sin \left(\frac{\pi}{2} \beth_{\partial_{g}}\right)\right)^{\ell_{g}}, \\
\left.1-\prod_{g=1}^{n}\left(\sin \left(\frac{\pi}{2} 1-\right\rceil_{\partial_{g}}\right)\right)^{\ell_{g}}, \\
1-\prod_{g=1}^{n}\left(\sin \left(\frac{\pi}{2} 1-\beth_{\partial_{g}}\right)\right)^{\ell_{g}}
\end{array}\right)
\end{aligned}
$$

Proof. We prove Theorem 16, by employing mathematical induction on $n$. As for each $g$, $\left.\partial_{g}=\left\{\beth_{\partial_{g}}(\hbar),\right\rceil_{\partial_{g}}(\hbar), \beth_{\partial_{g}}(\hbar)\right\} \in \operatorname{SVNN}(\aleph)$, which implies that $\left.\beth_{\partial_{g^{\prime}}},\right\rceil_{\partial_{g^{\prime}}} \beth_{\partial_{g}} \in[0,1]$ and $\left.\beth_{\partial_{g}}+\right\urcorner_{\partial_{g}}+\beth_{\partial_{g}} \leq 3$. Then, the following steps of the mathematical induction have been executed.

Step-1: For $n=2$, we get

$$
S T-S V N W G\left(\partial_{1}, \partial_{2}\right)=\left(\sin \left(\partial_{1}\right)\right)^{\ell_{1}} \otimes\left(\sin \left(\partial_{2}\right)\right)^{\ell_{2}} .
$$

As by the Definition $12, \sin \left(\partial_{1}\right)$ and $\sin \left(\partial_{2}\right)$ are SFNs, and therefore $\left(\sin \left(\partial_{1}\right)\right)^{\ell_{1}} \nabla\left(\sin \left(\partial_{2}\right)\right)^{\ell_{2}}$ is also SVNN. Further, for $\partial_{1}$ and $\partial_{2}$, we have

$$
\begin{aligned}
& \operatorname{ST}-\operatorname{SVNWG}\left(\partial_{1}, \partial_{2}\right) \\
= & \left(\sin \left(\partial_{1}\right)\right)^{\ell_{1}} \otimes\left(\sin \left(\partial_{2}\right)\right)^{\ell_{2}} \\
= & \left(\begin{array}{c}
\left(\sin \left(\frac{\pi}{2} \beth_{\partial_{1}}\right)\right)^{\ell_{1}}, \\
1-\left(\sin \left(\frac{\pi}{2} 1-\beth_{\partial_{1}}\right)\right)^{\ell_{1}}, \\
1-\left(\sin \left(\frac{\pi}{2} 1-\beth_{\partial_{1}}\right)\right)^{\ell_{1}}
\end{array}\right) \boxplus\left(\begin{array}{c}
\left(\sin \left(\frac{\pi}{2} \beth_{\partial_{2}}\right)\right)^{\ell_{2}}, \\
\left.1-\left(\sin \left(\frac{\pi}{2} 1-\right\rceil_{\partial_{2}}\right)\right)^{\ell_{2}}, \\
1-\left(\sin \left(\frac{\pi}{2} 1-\beth_{\partial_{2}}\right)\right)^{\ell_{2}}
\end{array}\right) \\
= & \left(\begin{array}{c}
\prod_{g=1}^{2}\left(\sin \left(\frac{\pi}{2} \beth_{\partial_{g}}\right)\right)^{\ell_{g}}, \\
\left.1-\prod_{g=1}^{2}\left(\sin \left(\frac{\pi}{2} 1-\right\rceil_{\partial_{g}}\right)\right)^{\ell_{g}}, \\
1-\prod_{g=1}^{2}\left(\sin \left(\frac{\pi}{2} 1-\beth_{\partial_{g}}\right)\right)^{\ell_{g}}
\end{array}\right)
\end{aligned}
$$


Step-2: Suppose that Equation (3) is holds for $n=\kappa$, we have

$$
S T-\operatorname{SVNWG}\left(\partial_{1}, \partial_{2}, \ldots \partial_{\kappa}\right)=\left(\begin{array}{c}
\prod_{g=1}^{\kappa}\left(\sin \left(\frac{\pi}{2} \beth_{\partial_{g}}\right)\right)^{\ell_{g}}, \\
\left.1-\prod_{g=1}^{\kappa}\left(\sin \left(\frac{\pi}{2} 1-\right\rceil_{\partial_{g}}\right)\right)^{\ell_{g}}, \\
1-\prod_{g=1}^{\kappa}\left(\sin \left(\frac{\pi}{2} 1-\beth_{\partial_{g}}\right)\right)^{\ell_{g}}
\end{array}\right)
$$

Step-3: Now we have to prove that Equation (3) is holds for $n=\kappa+1$.

$$
\begin{aligned}
& S T-S V N W G\left(\partial_{1}, \partial_{2}, \ldots \partial_{\kappa+1}\right) \\
= & \prod_{g=1}^{\kappa}\left(\sin \left(\partial_{g}\right)\right)^{\ell_{g}} \otimes\left(\sin \left(\partial_{\kappa+1}\right)\right)^{\ell_{\kappa+1}} \\
= & \left(\begin{array}{c}
\prod_{g=1}^{\kappa}\left(\sin \left(\frac{\pi}{2} \beth_{\partial_{g}}\right)\right)^{\ell_{g}}, \\
\left.1-\prod_{g=1}^{\kappa}\left(\sin \left(\frac{\pi}{2} 1-\right\rceil_{\partial_{g}}\right)\right)^{\ell_{g}}, \\
1-\prod_{g=1}^{\kappa}\left(\sin \left(\frac{\pi}{2} 1-\beth_{\partial_{g}}\right)\right)^{\ell_{g}}
\end{array}\right) \otimes\left(\begin{array}{c}
\left(\sin \left(\frac{\pi}{2} \beth_{\partial_{\kappa+1}}\right)\right)^{\ell_{\kappa+1}}, \\
\left.1-\left(\sin \left(\frac{\pi}{2} 1-\right\rceil_{\partial_{\kappa+1}}\right)\right)^{\ell_{\kappa+1}}, \\
1-\left(\sin \left(\frac{\pi}{2} 1-\beth_{\partial_{\kappa+1}}\right)\right)^{\ell_{\kappa+1}}
\end{array}\right) \\
= & \left(\begin{array}{c}
\prod_{g=1}^{\kappa+1}\left(\sin \left(\frac{\pi}{2} \beth_{\partial_{g}}\right)\right)^{\ell_{g}}, \\
\left.1-\prod_{g=1}^{\kappa+1}\left(\sin \left(\frac{\pi}{2} 1-\right\rceil_{\partial_{g}}\right)\right)^{\ell_{g}}, \\
1-\prod_{g=1}^{\kappa+1}\left(\sin \left(\frac{\pi}{2} 1-\beth_{\partial_{g}}\right)\right)^{\ell_{g}}
\end{array}\right)
\end{aligned}
$$

that is, when $n=z+1$, Equation (3) also holds.

Therefore, Equation (3) holds for any $n$. The proof is completed.

Theorem 17. (Idempotancy) Let $\left.\partial_{g}=\left\{\beth_{\partial_{g}}(\hbar),\right\rceil_{\partial_{g}}(\hbar), \beth_{\partial_{g}}(\hbar)\right\} \in \operatorname{SVNN}(\aleph)(g=1,2,3, \ldots, n)$ such that $\partial_{g}=\partial$. Then,

$$
S T-\operatorname{SVNWG}\left(\partial_{1}, \partial_{2}, \ldots, \partial_{n}\right)=\sin (\partial) \text {. }
$$

Theorem 18. (Boundedness) Let $\left.\partial_{g}=\left\{\beth_{\partial_{g}}(\hbar),\right\rceil_{\partial_{g}}(\hbar), \beth_{\partial_{g}}(\hbar)\right\}, \partial_{g}^{-}=$ $\left\{\min \left(\beth_{\partial_{g}}(\hbar)\right), \max \left(\nearrow_{\partial_{g}}(\hbar)\right), \max \left(\beth_{\partial_{g}}(\hbar)\right)\right\}$ and $\partial_{g}^{+}=\left\{\max \left(\beth_{\partial_{g}}(\hbar)\right), \min \left(\nearrow_{\partial_{g}}(\hbar)\right), \min \left(\beth_{\partial_{g}}(\hbar)\right)\right\}$ $\in \operatorname{SVNN}(\aleph)(g=1,2,3, \ldots, n)$. Then,

$$
\sin \left(\partial_{g}^{-}\right) \leq S T-S V N W G\left(\partial_{1}, \partial_{2}, \ldots, \partial_{n}\right) \leq \sin \left(\partial_{g}^{+}\right) .
$$

Theorem 19. (Monotonically) Let $\left.\left.\partial_{g}=\left\{\beth_{\partial_{g}}(\hbar),\right\rceil_{\partial_{g}}(\hbar), \beth_{\partial_{g}}(\hbar)\right\}, \partial_{g}^{*}=\left\{\beth_{\partial_{g}}^{*}(\hbar),\right\rceil_{\partial_{g}}^{*}(\hbar), \beth_{\partial_{g}}^{*}(\hbar)\right\} \in$ $\operatorname{SVNN}(\aleph)(g=1,2,3, \ldots, n)$. If $\left.\left.\beth_{\partial_{g}} \leq \beth_{\partial_{g^{\prime}}}^{*}\right\rceil_{\partial_{g}} \leq\right\rceil_{\partial_{g}}^{*}$ and $\beth_{\partial_{g}} \leq \beth_{\partial_{g^{\prime}}}^{*}$ then

$$
S T-\operatorname{SVNWG}\left(\partial_{1}, \partial_{2}, \ldots, \partial_{n}\right) \leq S T-\operatorname{SVNWG}\left(\partial_{1}^{*}, \partial_{2}^{*}, \ldots, \partial_{n}^{*}\right) .
$$

Theorem 20. (Symmetric) Let $\left.\partial_{g}=\left\{\beth_{\partial_{g}}(\hbar),\right\rceil_{\partial_{g}}(\hbar), \beth_{\partial_{g}}(\hbar)\right\}, \partial_{g}^{*}=\left\{\beth_{\partial_{g}^{*}}^{*}(\hbar), \neg_{\partial_{g}^{*}}^{*}(\hbar), \beth_{\partial_{g}}^{*}(\hbar)\right\} \in$ $\operatorname{SVNN}(\aleph)(g=1,2,3, \ldots, n)$. Then

$$
S T-\operatorname{SVNWG}\left(\partial_{1}, \partial_{2}, \ldots, \partial_{n}\right)=S T-S V N W G\left(\partial_{1}^{*}, \partial_{2}^{*}, \ldots, \partial_{n}^{*}\right),
$$

whenever $\partial_{g}^{*}(g=1,2,3, \ldots, n)$ is any version of $\partial_{g}(g=1,2,3, \ldots, n)$.

Proof of above theorems are follows from Theorems 7-10 similarly. 
Definition 19. Let $\left.\partial_{g}=\left\{\beth_{\partial_{g}}(\hbar),\right\rceil_{\partial_{g}}(\hbar), \beth_{\partial_{g}}(\hbar)\right\} \in \operatorname{SVNN}(\aleph)(g=1,2,3, \ldots, n)$. Then, the sine trigonometric ordered weighted geometric aggregation operator for $S V N N(\aleph)$ is denoted by ST - SVNOWG and defined as follows,

$$
\begin{aligned}
\operatorname{ST}-\operatorname{SVNOWG}\left(\partial_{1}, \partial_{2}, \ldots, \partial_{n}\right) & =\left(\sin \left(\partial_{v(1)}\right)\right)^{\ell_{1}} \otimes\left(\sin \left(\partial_{v(2)}\right)\right)^{\ell_{2}} \otimes \ldots \otimes\left(\sin \left(\partial_{v(n)}\right)\right)^{\ell_{n}} \\
& =\prod_{g=1}^{n}\left(\sin \left(\partial_{v(g)}\right)\right)^{\ell_{g}}
\end{aligned}
$$

where $v(g)$ is denoted for ordered and $(v(1), v(2), v(3), \ldots, v(n))$ is a permutation of $(1,2,3, \ldots, n)$, subject to $\varepsilon_{v(g-1)} \geq \varepsilon_{v(g)}$ for all $g$. Also, $\ell_{g}(g=1,2, \ldots, n)$ represents the weights of $\partial_{g}(g=1,2,3, \ldots, n)$ with $\ell_{g} \geq 0$ and $\sum_{g=1}^{n} \ell_{g}=1$.

Theorem 21. Let $\left.\partial_{g}=\left\{\beth_{\partial_{g}}(\hbar),\right\urcorner_{\partial_{g}}(\hbar), \beth_{\partial_{g}}(\hbar)\right\} \in S V N N(\aleph)(g=1,2,3, \ldots, n)$ and the weight vector of $\partial_{g}(g=1,2,3, \ldots, n)$ be denoted by $\ell=\left(\ell_{1}, \ell_{2}, \ldots, \ell_{n}\right)^{T}$ subject to $\sum_{g=1}^{n} \ell_{g}=1$. The ST $-S V N O W G$ operator is a mapping $G^{n} \longrightarrow G$ such that

$$
\begin{aligned}
\operatorname{ST}-\operatorname{SVNOWG}\left(\partial_{1}, \partial_{2}, \ldots, \partial_{n}\right)= & \prod_{g=1}^{n}\left(\sin \left(\partial_{v(g)}\right)\right)^{\ell_{g}} \\
& =\left(\begin{array}{c}
\prod_{g=1}^{n}\left(\sin \left(\frac{\pi}{2} \beth_{\partial_{v(g)}}\right)\right)^{\ell_{g}}, \\
\left.1-\prod_{g=1}^{n}\left(\sin \left(\frac{\pi}{2} 1-\right\rceil_{\partial_{v(g)}}\right)\right)^{\ell_{g}}, \\
1-\prod_{g=1}^{n}\left(\sin \left(\frac{\pi}{2} 1-\beth_{\partial_{v(g)}}\right)\right)^{\ell_{g}}
\end{array}\right)
\end{aligned}
$$

Proof. Follows from Theorem 16 similarly.

Theorem 22. (Idempotancy) Let $\left.\partial_{g}=\left\{\beth_{\partial_{g}}(\hbar),\right\rceil_{\partial_{g}}(\hbar), \beth_{\partial_{g}}(\hbar)\right\} \in \operatorname{SVNN}(\aleph)(g=1,2,3, \ldots, n)$ such that $\partial_{g}=\partial$. Then,

$$
S T-\operatorname{SVNOWG}\left(\partial_{1}, \partial_{2}, \ldots, \partial_{n}\right)=\sin (\partial) \text {. }
$$

Theorem 23. (Boundedness) Let $\left.\partial_{g}=\left\{\beth_{\partial_{g}}(\hbar),\right\urcorner_{\partial_{g}}(\hbar), \beth_{\partial_{g}}(\hbar)\right\}, \partial_{g}^{-}=$ $\left\{\min \left(\beth_{\partial_{g}}(\hbar)\right), \max \left(\nearrow_{\partial_{g}}(\hbar)\right), \max \left(\beth_{\partial_{g}}(\hbar)\right)\right\}$ and $\partial_{g}^{+}=\left\{\max \left(\beth_{\partial_{g}}(\hbar)\right), \min \left(\nearrow_{\partial_{g}}(\hbar)\right), \min \left(\beth_{\partial_{g}}(\hbar)\right)\right\}$ $\in \operatorname{SVNN}(\aleph)(g=1,2,3, \ldots, n)$. Then,

$$
\sin \left(\partial_{g}^{-}\right) \leq S T-S V N O W G\left(\partial_{1}, \partial_{2}, \ldots, \partial_{n}\right) \leq \sin \left(\partial_{g}^{+}\right) .
$$

Theorem 24. (Monotonically) Let $\left.\left.\partial_{g}=\left\{\beth_{\partial_{g}}(\hbar),\right\rceil_{\partial_{g}}(\hbar), \beth_{\partial_{g}}(\hbar)\right\}, \partial_{g}^{*}=\left\{\beth_{\partial_{g}}^{*}(\hbar),\right\rceil_{\partial_{g}}^{*}(\hbar), \beth_{\partial_{g}}^{*}(\hbar)\right\} \in$ $\operatorname{SVNN}(\aleph)(g=1,2,3, \ldots, n)$. If $\left.\left.\beth_{\partial_{g}} \leq \beth_{\partial_{g^{\prime}}^{*}}^{*}\right\urcorner_{\partial_{g}} \leq\right\rceil_{\partial_{g}^{*}}^{*}$ and $\beth_{\partial_{g}} \leq \beth_{\partial_{g^{\prime}}^{*}}^{*}$, then

$$
S T-\operatorname{SVNOWG}\left(\partial_{1}, \partial_{2}, \ldots, \partial_{n}\right) \leq S T-\operatorname{SVNOWG}\left(\partial_{1}^{*}, \partial_{2}^{*}, \ldots, \partial_{n}^{*}\right) \text {. }
$$

Theorem 25. (Symmetric) Let $\left.\partial_{g}=\left\{\beth_{\partial_{g}}(\hbar),\right\rceil_{\partial_{g}}(\hbar), \beth_{\partial_{g}}(\hbar)\right\}, \partial_{g}^{*}=\left\{\beth_{\partial_{g}^{*}}^{*}(\hbar), \neg_{\partial_{g}^{*}}^{*}(\hbar), \beth_{\partial_{g}}^{*}(\hbar)\right\} \in$ $\operatorname{SVNN}(\aleph)(g=1,2,3, \ldots, n)$. Then

$$
S T-\operatorname{SVNOWG}\left(\partial_{1}, \partial_{2}, \ldots, \partial_{n}\right)=S T-\operatorname{SVNOWG}\left(\partial_{1}^{*}, \partial_{2}^{*}, \ldots, \partial_{n}^{*}\right),
$$

whenever $\partial_{g}^{*}(g=1,2,3, \ldots, n)$ is any version of $\partial_{g}(g=1,2,3, \ldots, n)$. 
Proof of above theorems follows from Theorems 7-10 similarly.

\subsection{Fundamental Properties of the Proposed AOs}

In this section, we investigated the several relations between the proposed AOs and study their some fundamental properties as follows.

Theorem 26. Let $\left.\partial_{g}=\left\{\beth_{\partial_{g}}(\hbar),\right\rceil_{\partial_{g}}(\hbar), \beth_{\partial_{g}}(\hbar)\right\} \in \operatorname{SVNN}(\aleph)(g=1,2)$. Then, we have

$$
\sin \left(\partial_{1}\right) \boxplus \sin \left(\partial_{2}\right) \geq \sin \left(\partial_{1}\right) \otimes \sin \left(\partial_{2}\right)
$$

Proof. Since, $\partial_{g} \in S V N N(\aleph)(g=1,2)$. Then, by using Definition 13, we have

$$
\sin \left(\partial_{1}\right) \boxplus \sin \left(\partial_{2}\right)=\left(\begin{array}{c}
1-\left(1-\sin \left(\frac{\pi}{2} \beth_{\partial_{1}}\right)\right)\left(1-\sin \left(\frac{\pi}{2} \beth_{\partial_{2}}\right)\right), \\
\left(1-\sin \left(\frac{\pi}{2} 1-\beth_{\partial_{1}}\right)\right)\left(1-\sin \left(\frac{\pi}{2} 1-\beth_{\partial_{2}}\right)\right), \\
\left(1-\sin \left(\frac{\pi}{2} 1-\beth_{\partial_{1}}\right)\right)\left(1-\sin \left(\frac{\pi}{2} 1-\beth_{\partial_{2}}\right)\right)
\end{array}\right)
$$

and

$$
\sin \left(\partial_{1}\right) \otimes \sin \left(\partial_{2}\right)=\left(\begin{array}{c}
\sin \left(\frac{\pi}{2} \beth_{\partial_{1}}\right) \sin \left(\frac{\pi}{2} \beth_{\partial_{2}}\right), \\
1-\left(\sin \left(\frac{\pi}{2} 1-\beth_{\partial_{1}}\right)\right)\left(\sin \left(\frac{\pi}{2} 1-\beth_{\varkappa 2}\right)\right), \\
1-\left(\sin \left(\frac{\pi}{2} 1-\beth_{\partial_{1}}\right)\right)\left(\sin \left(\frac{\pi}{2} 1-\beth_{\varkappa 2}\right)\right)
\end{array}\right)
$$

As for any two non-negative real numbers $l$ and $m$, their arithmetic mean is greater than or equal to their geometric mean, $\frac{l+m}{2} \geq l m \Rightarrow l+m-l m \geq l m \Rightarrow 1-(1-l)(1-m) \geq l m$. Thus, by taking $l=\sin \left(\frac{\pi}{2} \beth_{\partial_{1}}\right)$ and $m=\sin \left(\frac{\pi}{2} \beth_{\partial_{2}}\right)$ we have $1-\left(1-\sin \left(\frac{\pi}{2} \beth_{\partial_{1}}\right)\right)\left(1-\sin \left(\frac{\pi}{2} \beth_{\partial_{2}}\right)\right) \geq$ $\sin \left(\frac{\pi}{2} \beth_{\partial_{1}}\right) \sin \left(\frac{\pi}{2} \beth_{\partial_{2}}\right)$, which implies that

$$
1-\left(1-\sin \left(\frac{\pi}{2} \beth_{\partial_{1}}\right)\right)\left(1-\sin \left(\frac{\pi}{2} \beth_{\partial_{2}}\right)\right) \geq \sin \left(\frac{\pi}{2} \beth_{\partial_{1}}\right) \sin \left(\frac{\pi}{2} \beth_{\partial_{2}}\right)
$$

Similarly, we have

$$
\left.\left.\left.\left.\left(1-\sin \left(\frac{\pi}{2} 1-\right\urcorner_{\partial_{1}}\right)\right)\left(1-\sin \left(\frac{\pi}{2} 1-\right\urcorner_{\partial_{2}}\right)\right) \leq 1-\left(\sin \left(\frac{\pi}{2} 1-\right\urcorner_{\partial_{1}}\right)\right)\left(\sin \left(\frac{\pi}{2} 1-\right\urcorner_{\varkappa 2}\right)\right)
$$

and

$$
\left(1-\sin \left(\frac{\pi}{2} 1-\beth_{\partial_{1}}\right)\right)\left(1-\sin \left(\frac{\pi}{2} 1-\beth_{\partial_{2}}\right)\right) \leq 1-\left(\sin \left(\frac{\pi}{2} 1-\beth_{\partial_{1}}\right)\right)\left(\sin \left(\frac{\pi}{2} 1-\beth_{\varkappa 2}\right)\right)
$$

Therefore,

$$
\sin \left(\partial_{1}\right) \boxplus \sin \left(\partial_{2}\right) \geq \sin \left(\partial_{1}\right) \otimes \sin \left(\partial_{2}\right)
$$

Proved.

Theorem 27. Let $\left.\partial=\left\{\beth_{\partial}(\hbar),\right\rceil_{\partial}(\hbar), \beth_{\partial}(\hbar)\right\} \in \operatorname{SVNN}(\aleph)$ and $\psi \geq 0$ be any real number, then

(1) $\psi \sin (\partial) \geq(\sin (\partial))^{\psi}$ iff $\psi \geq 1$,

(2) $\psi \sin (\partial) \leq(\sin (\partial))^{\psi}$ iff $0<\psi \leq 1$.

Proof. Follows from Theorem 26, similarly.

Lemma 1. For $l_{g} \geq 0$ and $m_{g} \geq 0$, then we have $\prod_{g=1}^{n}\left(l_{g}\right)^{m_{g}} \leq \sum_{g=1}^{n} m_{g} l_{g}$ and if $l_{1}=l_{2}=\ldots=l_{n}$ then equality holds.

Lemma 2. Let $0 \leq l, m \leq 1$, and $0 \leq x \leq 1$, then $0 \leq l x+m(1-x) \leq 1$.

Lemma 3. Let $0 \leq l, m \leq 1$, then $\sqrt{1-\left(1-l^{2}\right)\left(1-m^{2}\right)} \geq l m$. 
Theorem 28. Let $\left.\partial_{g}=\left\{\beth_{\partial_{g}}(\hbar),\right\rceil_{\partial_{g}}(\hbar), \beth_{\partial_{g}}(\hbar)\right\} \in \operatorname{SVNN}(\aleph)(g=1,2,3, \ldots, n)$. Then,

$$
S T-S V N W A\left(\partial_{1}, \partial_{2}, \ldots, \partial_{n}\right) \geq S T-S V N W G\left(\partial_{1}, \partial_{2}, \ldots, \partial_{n}\right)
$$

where equality holds iff $\partial_{1}=\partial_{2}=\ldots=\partial_{n}$.

Proof. Follows from Theorem 26, similarly.

\section{Decision-Making Technique}

This part presents a decision-making methodology, followed by an illustrative example, to solve decision-making problems (DMPs) under SVNS setting. Multi-attribute decision-making issues can be demonstrated in the form of a decision matrix, in which the columns reflect the set of attributes and the rows are alternatives [59-63]. Thus, for decision matrix $D_{n \times m}$, consider a set of $n$ alternatives $\left\{\aleph_{1}, \aleph_{2}, \aleph_{3}, \ldots, \aleph_{n}\right\}$ and $m$ criteria/attributes $\left\{t_{1}, t_{2}, t_{3}, \ldots, t_{m}\right\}$. The unknown weight vector of $m$ criteria/attributes is denoted by $W=\left\{\kappa_{1}, \kappa_{2}, \kappa_{3}, \ldots, \kappa_{m}\right\}$ with subject to $\ell_{g} \in[0,1]$ such that $\sum_{g=1}^{m} \ell_{g}=1$.

Suppose that the single-valued neutrosophic decision matrix is denoted by $D=\left(\partial_{i j}\right)_{n \times m}=$ $\left.\left\langle\beth_{i j},\right\urcorner_{i j}, \beth_{i j}\right\rangle_{n \times m}$, where $\beth_{i j}$ represents the truth degree of the alternative gratifies the criteria $t_{j}$ considered by decision-maker (DM), $\urcorner_{i j}$ represents the degree of the alternative is indeterminacy for the criteria $t_{j}$ considered by decision maker $(\mathrm{DM})$, and $\beth_{i j}$ represents the degree of the alternative does not gratify the criteria $t_{j}$ considered by decision-maker (DM). The algorithm consists of the following steps.

Step-1 Summarize the values of each alternative in term of decision matrix $D^{(k)}=\left(\partial_{i j}^{(k)}\right)_{n \times m}$ with SVNS information.

Step-2 Construct the normalized decision matrix $P=\left(p_{i j}\right)$ from $D=\left(\partial_{i j}\right)$, where $p_{i j}$ is calculated as

$$
p_{i j}= \begin{cases}\left.\left(\beth_{i j},\right\urcorner_{i j}, \beth_{i j}\right) & \text { If criteria are benefit type } \\ \left(\beth_{i j}, \neg_{i j}, \beth_{i j}\right) & \text { If criteria are cost type }\end{cases}
$$

Step-3 Calculate the aggregate information of the decision-makers information either SFWA/SFWG operator.

$$
\left.\operatorname{SVNWA}\left(\partial_{1}, \partial_{2}, \ldots, \partial_{n}\right)=\left\{1-\Pi_{g=1}^{n}\left(1-\beth_{\partial_{g}}\right)^{\ell_{g}}, \Pi_{g=1}^{n}(\rceil_{\partial_{g}}\right)^{\ell_{g}}, \Pi_{g=1}^{n}\left(\beth_{\partial_{g}}\right)^{\ell_{g}}\right\}
$$

or

$$
\left.\operatorname{SVNWG}\left(\partial_{1}, \partial_{2}, \ldots, \partial_{n}\right)=\left\{\Pi_{g=1}^{n}\left(\beth_{\partial_{g}}\right)^{\ell_{g}}, 1-\Pi_{g=1}^{n}(1-\urcorner_{\partial_{g}}\right)^{\ell_{g}}, 1-\Pi_{g=1}^{n}\left(1-\beth_{\partial_{g}}\right)^{\ell_{g}}\right\}
$$

Step-4 If the attribute weights are known as a prior then utilize them. Otherwise, we compute them by utilizing the concept of the entropy measure. For it, the information of criteria $t_{j}$ based on entropy measure is computed as

$$
\left.\left.E_{j}(\partial)=\frac{1}{(\sqrt{2}-1) m} \sum_{i=1}^{m}\left[\sin \left(\frac{\pi}{4}\left(1+\beth_{\partial_{i j}}-\right\urcorner_{\partial_{i j}}-\beth_{\partial_{i j}}\right)\right)+\sin \left(\frac{\pi}{4}\left(1-\beth_{\partial_{i j}}+\right\rceil_{\partial_{i j}}+\beth_{\partial_{i j}}\right)\right)-1\right]
$$

where $\frac{1}{(\sqrt{2}-1) m}$ is a constant for assuring $0 \leq E_{j}(\partial) \leq 1$.

Step-5 Using proposed sine trigonometric aggregation operators and attributes weight vector, the collective single-valued neutrosophic information of the each alternative $\left\{\aleph_{1}, \aleph_{2}, \aleph_{3}, \ldots, \aleph_{n}\right\}$ are obtained. 
Step-6 Evaluate the scores values $\overline{s c}(\partial)$ of collective single-valued neutrosophic numbers and rank according the maximum score values. If the score values of two $\partial_{1}$ and $\partial_{2}$ are same, then find the accuracy degrees $\underline{a c}\left(\partial_{1}\right)$ and $\underline{a c}\left(\partial_{2}\right)$, respectively, then we rank the $\partial_{1}$ and $\partial_{2}$ according the maximum degree.

Step-7 Select the optimal alternative according the maximum score value or accuracy degree.

\section{Application of Proposed Decision-Making Technique}

In this section, a numerical application about hydrogen power plant selection problem is firstly used to illustrate the designed decision-making method. Then, a comparison between the presented sine trigonometric aggregation operators and the existing aggregation operators of SVNNs are carried out to demonstrate the characteristic and benefit of the presented AOs.

\subsection{Practical Case Study}

Maximizing the reach of technologies and the efficient use of renewable resources has always been a key task for developing sustainable and environmentally friendly energy with a view to future prospects. Invariably, in dealing with all renewable energy projects, the problem of site selection is always a very important one, where experts and decision-makers take all possible qualitative and quantitative factors into account. In particular, selecting the right location for the hydrogen power plant project is an important task that is consistently addressed through a multi-criteria decision-making process. Hydrogen energy is one of the most efficient and cleanest energy sources that contribute significantly to the share of energy in the world.

The sites under consideration must have been chosen through professional communication by the competent experts. All the attributes affecting the site selection have been determined on the basis of the expert's/decision-maker's opinion and the available literature. For the sake of selecting the best site/location, the decision-makers must take the social aspects, environment aspects, technology aspects, financial implications, and also some major characteristic aspects. We take a case study for this selection problem in a conventional frame where there are five available sites, say, $S_{1}, S_{2}, S_{3}, S_{4}$ and $S_{5}$, which are under consideration in solving the problem. These sites have been systematically examined with respect to the five main attributes, say, $f_{1}$ (Social Aspect) , $f_{2}$ (Environment Aspect), $f_{3}$ (Technology Aspect), $f_{4}$ (Economical Aspect), and $f_{5}$ (Site Characteristics). Naturally, a better solution is expected if the number of attributes are increased. The problem of selecting the best possible hydrogen power plant site from the available set of alternatives is being mathematically and critically solved under the expert's/decision-maker's opinion and criteria weights taking the single-valued neutrosophic environment. Due to the fuzziness and uncertainty of the experts' cognition, they cannot provide the complete decision information, and the evaluation information is shown in the following Table 1. In this evaluation, the expert was asked to use SVN information and attributes weights are $(0.15,0.28,0.20,0.22,0.15)^{T}$.

Step-1 Information result of the expert is listed in Table 1;

Table 1. SVN Information (D).

\begin{tabular}{cccccc}
\hline & $f_{1}$ & $f_{2}$ & $f_{3}$ & $f_{4}$ & $f_{5}$ \\
\hline$S_{1}$ & $(0.5,0.3,0.4)$ & $(0.3,0.2,0.5)$ & $(0.2,0.2,0.6)$ & $(0.4,0.2,0.3)$ & $(0.3,0.3,0.4)$ \\
$S_{2}$ & $(0.7,0.1,0.3)$ & $(0.3,0.2,0.7)$ & $(0.6,0.3,0.2)$ & $(0.2,0.4,0.6)$ & $(0.7,0.1,0.2)$ \\
$S_{3}$ & $(0.5,0.3,0.4)$ & $(0.4,0.2,0.6)$ & $(0.6,0.1,0.2)$ & $(0.3,0.1,0.5)$ & $(0.6,0.4,0.3)$ \\
$S_{4}$ & $(0.7,0.3,0.2)$ & $(0.2,0.2,0.7)$ & $(0.4,0.5,0.2)$ & $(0.2,0.2,0.5)$ & $(0.4,0.5,0.4)$ \\
$S_{5}$ & $(0.4,0.1,0.3)$ & $(0.2,0.1,0.5)$ & $(0.4,0.1,0.5)$ & $(0.6,0.3,0.4)$ & $(0.3,0.2,0.4)$ \\
\hline
\end{tabular}

Step-2 According to the expert, attributes $t_{1}, t_{3}$, and $t_{5}$ are benefits type, $t_{2}$ and $t_{4}$ are cost attributes. Normalized matrix computed as given formula 5, and results are shown in Table 2; 
Table 2. Normalized SVN information $(P)$.

\begin{tabular}{cccccc}
\hline & $f_{1}$ & $f_{2}$ & $f_{3}$ & $f_{4}$ & $f_{5}$ \\
\hline$S_{1}$ & $(0.5,0.3,0.4)$ & $(0.5,0.2,0.3)$ & $(0.2,0.2,0.6)$ & $(0.3,0.2,0.4)$ & $(0.3,0.3,0.4)$ \\
$S_{2}$ & $(0.7,0.1,0.3)$ & $(0.7,0.2,0.3)$ & $(0.6,0.3,0.2)$ & $(0.6,0.4,0.2)$ & $(0.7,0.1,0.2)$ \\
$S_{3}$ & $(0.5,0.3,0.4)$ & $(0.6,0.2,0.4)$ & $(0.6,0.1,0.2)$ & $(0.5,0.1,0.3)$ & $(0.6,0.4,0.3)$ \\
$S_{4}$ & $(0.7,0.3,0.2)$ & $(0.7,0.2,0.2)$ & $(0.4,0.5,0.2)$ & $(0.5,0.2,0.2)$ & $(0.4,0.5,0.4)$ \\
$S_{5}$ & $(0.4,0.1,0.3)$ & $(0.5,0.1,0.2)$ & $(0.4,0.1,0.5)$ & $(0.4,0.3,0.6)$ & $(0.3,0.2,0.4)$ \\
\hline
\end{tabular}

Step-3 In this practical case study, only one expert (decision-maker) is involved, so here we do not need to compute the aggregated decision matrix.

Step-4 Known criteria weight vector is:

$$
\kappa=\left\{\kappa_{1}=0.15, \kappa_{2}=0.28, \kappa_{3}=0.20, \kappa_{4}=0.22, \kappa_{5}=0.15\right\}
$$

Step-5 Based on the weight vector and utilizing the proposed sine trigonometric AOs, the aggregated single-valued neutrosophic information of each alternatives are obtained in Table 3:

Table 3. Aggregated single-valued neutrosophic information.

\begin{tabular}{ccccc}
\hline & $S T-S V N W A$ & $S T-S V N O W A$ & $S T-S V N W G$ & $S T-S V N O W G$ \\
\hline$S_{1}$ & $(0.562,0.025,0.078)$ & $(0.567,0.029,0.081)$ & $(0.508,0.027,0.089)$ & $(0.518,0.032,0.088)$ \\
$S_{2}$ & $(0.862,0.020,0.028)$ & $(0.865,0.016,0.029)$ & $(0.855,0.033,0.030)$ & $(0.859,0.028,0.031)$ \\
$S_{3}$ & $(0.776,0.015,0.048)$ & $(0.770,0.016,0.048)$ & $(0.769,0.026,0.054)$ & $(0.763,0.030,0.053)$ \\
$S_{4}$ & $(0.784,0.042,0.024)$ & $(0.782,0.048,0.024)$ & $(0.732,0.060,0.029)$ & $(0.729,0.065,0.029)$ \\
$S_{5}$ & $(0.609,0.009,0.064)$ & $(0.583,0.009,0.067)$ & $(0.595,0.016,0.088)$ & $(0.570,0.014,0.084)$ \\
\hline
\end{tabular}

Step-6 Compute the score value of the each aggregated single-valued neutrosophic information of each alternative as follows in Table 4.

Table 4. Score values.

\begin{tabular}{cccccc}
\hline & $\overline{s c}\left(S_{1}\right)$ & $\overline{s c}\left(S_{2}\right)$ & $\overline{s c}\left(S_{3}\right)$ & $\overline{s c}\left(S_{4}\right)$ & $\overline{s c}\left(S_{5}\right)$ \\
\hline ST - SVNWA & 0.45785 & 0.81292 & 0.71225 & 0.71723 & 0.53521 \\
ST - SVNOWA & 0.45577 & 0.82036 & 0.70536 & 0.70931 & 0.50665 \\
ST-SVNWG & 0.39152 & 0.79117 & 0.68839 & 0.64236 & 0.49041 \\
ST - SVNOWG & 0.39711 & 0.79969 & 0.67968 & 0.63447 & 0.47160 \\
\hline
\end{tabular}

Step-7 Select the optimal alternative according the maximum score value given in Table 5 .

Table 5. Ranking.

\begin{tabular}{clc}
\hline & \multicolumn{1}{c}{ Score Ranking } & Best Alternative \\
\hline$S T-S V N W A$ & $\overline{S C}\left(S_{2}\right)>\overline{S C}\left(S_{4}\right)>\overline{S C}\left(S_{3}\right)>\overline{S C}\left(S_{5}\right)>\overline{S C}\left(S_{1}\right)$ & $S_{2}$ \\
$S T-S V N O W A$ & $\overline{S C}\left(S_{2}\right)>\overline{S C}\left(S_{4}\right)>\overline{S C}\left(S_{3}\right)>\overline{S C}\left(S_{5}\right)>\overline{S C}\left(S_{1}\right)$ & $S_{2}$ \\
$S T-S V N W G$ & $\overline{S C}\left(S_{2}\right)>\overline{S C}\left(S_{3}\right)>\overline{S C}\left(S_{4}\right)>\overline{S C}\left(S_{5}\right)>\overline{S C}\left(S_{1}\right)$ & $S_{2}$ \\
$S T-S V N O W G$ & $\overline{S C}\left(S_{2}\right)>\overline{S C}\left(S_{3}\right)>\overline{S C}\left(S_{4}\right)>\overline{S C}\left(S_{5}\right)>\overline{S C}\left(S_{1}\right)$ & $S_{2}$ \\
\hline
\end{tabular}

In our case study, we aim to select the the right location for the hydrogen power plant according to five attributes: Social Aspect, Environment Aspect, Technology Aspect, Economical Aspect, and Site Characteristics. After implementing the designed algorithm steps to the collective data in the form of a single-valued neutrosophic set based on the novel sine trigonometric operational rules. Based on the above computational process, we can conclude that the alternative $S_{2}$ is the best among the others and therefore it is highly recommended to select for the task/plan that is required. 


\subsection{Verification and the Comparison Analysis}

In the following, we provides some suitable examples to show the feasibility as well as effectiveness of the proposed novel decision-making method and make a comparison with the existing studies.

To using existing methods and different aggregation operators to computed aggregated single-valued information information are shown in Tables 6 and 7.

Table 6. Average aggregated SVN information.

\begin{tabular}{ccccc}
\hline & SVNWA [64] & SVNOWA [64] & NWA [57] & SVNFWA [65] \\
\hline$S_{1}$ & $\{0.377,0.225,0.400\}$ & $\{0.382,0.244,0.407\}$ & $\{0.377,0.231,0.422\}$ & $\{0.375,0.226,0.401\}$ \\
$S_{2}$ & $\{0.661,0.205,0.238\}$ & $\{0.666,0.180,0.243\}$ & $\{0.661,0.242,0.244\}$ & $\{0.661,0.207,0.238\}$ \\
$S_{3}$ & $\{0.565,0.176,0.313\}$ & $\{0.559,0.183,0.312\}$ & $\{0.565,0.210,0.327\}$ & $\{0.565,0.177,0.314\}$ \\
$S_{4}$ & $\{0.572,0.292,0.221\}$ & $\{0.570,0.314,0.221\}$ & $\{0.572,0.334,0.233\}$ & $\{0.569,0.295,0.222\}$ \\
$S_{5}$ & $\{0.416,0.141,0.360\}$ & $\{0.396,0.137,0.369\}$ & $\{0.416,0.163,0.413\}$ & $\{0.415,0.142,0.364\}$ \\
\hline
\end{tabular}

Table 7. Average aggregated SVN information.

\begin{tabular}{ccccc}
\hline & SVNHWA [66] $\gamma=\mathbf{2}$ & SVNHWA [66] $\gamma=3$ & L-SVNWA [55] & L-SVNOWA [55] \\
\hline$S_{1}$ & $\{0.372,0.226,0.403\}$ & $\{0.369,0.226,0.404\}$ & $\{0.313,0.175,0.354\}$ & $\{0.322,0.192,0.360\}$ \\
$S_{2}$ & $\{0.660,0.208,0.238\}$ & $\{0.660,0.209,0.239\}$ & $\{0.648,0.198,0.231\}$ & $\{0.654,0.171,0.236\}$ \\
$S_{3}$ & $\{0.564,0.179,0.314\}$ & $\{0.564,0.180,0.315\}$ & $\{0.498,0.173,0.332\}$ & $\{0.489,0.182,0.330\}$ \\
$S_{4}$ & $\{0.566,0.297,0.223\}$ & $\{0.563,0.300,0.223\}$ & $\{0.558,0.273,0.194\}$ & $\{0.556,0.297,0.194\}$ \\
$S_{5}$ & $\{0.415,0.142,0.368\}$ & $\{0.414,0.143,0.371\}$ & $\{0.284,0.124,0.375\}$ & $\{0.244,0.120,0.383\}$ \\
\hline
\end{tabular}

Now, we analysis the ranking of the alternative according to their aggregated informations in Tables 8 and 9.

Table 8. Overall ranking of the alternatives.

\begin{tabular}{ccc}
\hline Existing Operators & Ranking & Best Alternative \\
\hline NWA [57] & $S_{2}>S_{3}>S_{4}>S_{5}>S_{1}$ & $S_{2}$ \\
SVNWA [64] & $S_{2}>S_{3}>S_{4}>S_{5}>S_{1}$ & $S_{2}$ \\
SVNOWA [64] & $S_{2}>S_{3}>S_{4}>S_{5}>S_{1}$ & $S_{2}$ \\
SVNWG [64] & $S_{2}>S_{3}>S_{4}>S_{5}>S_{1}$ & $S_{2}$ \\
SVNOWG [64] & $S_{2}>S_{3}>S_{4}>S_{5}>S_{1}$ & $S_{2}$ \\
SVNFWA [65] & $S_{2}>S_{3}>S_{4}>S_{5}>S_{1}$ & $S_{2}$ \\
SVNHWA [66] $\gamma=2$ & $S_{2}>S_{3}>S_{4}>S_{5}>S_{1}$ & $S_{2}$ \\
SVNHWA [66] $\gamma=3$ & $S_{2}>S_{3}>S_{4}>S_{5}>S_{1}$ & $S_{2}$ \\
NWG [45] & $S_{2}>S_{3}>S_{4}>S_{5}>S_{1}$ & $S_{2}$ \\
SVNFWG [65] & $S_{2}>S_{3}>S_{4}>S_{5}>S_{1}$ & $S_{2}$ \\
SVNHWG [66] $\gamma=2$ & $S_{2}>S_{3}>S_{4}>S_{5}>S_{1}$ & $S_{2}$ \\
SVNHWG [66] $\gamma=3$ & $S_{2}>S_{3}>S_{4}>S_{5}>S_{1}$ & $S_{2}$ \\
SNWEA [54] & $S_{2}>S_{3}>S_{5}>S_{4}>S_{1}$ & $S_{2}$ \\
L-SVNWA [55] & $S_{2}>S_{4}>S_{3}>S_{5}>S_{1}$ & $S_{2}$ \\
L-SVNOWA [55] & $S_{2}>S_{4}>S_{3}>S_{5}>S_{1}$ & $S_{2}$ \\
L-SVNWG [55] & $S_{2}>S_{4}>S_{3}>S_{1}>S_{5}$ & $S_{2}$ \\
L-SVNOWG [55] & $S_{2}>S_{3}>S_{4}>S_{5}>S_{1}$ & $S_{2}$ \\
\hline
\end{tabular}


Table 9. Overall ranking of the alternatives.

\begin{tabular}{ccc}
\hline Proposed Operators & Ranking & Best Alternative \\
\hline L-SVNWA & $S_{2}>S_{4}>S_{3}>S_{5}>S_{1}$ & $S_{2}$ \\
L-SVNWG & $S_{2}>S_{3}>S_{4}>S_{5}>S_{1}$ & $S_{2}$ \\
L-SVNOWA & $S_{2}>S_{4}>S_{3}>S_{5}>S_{1}$ & $S_{2}$ \\
L-SVNOWG & $S_{2}>S_{3}>S_{4}>S_{5}>S_{1}$ & $S_{2}$ \\
\hline
\end{tabular}

The bast alternative is $S_{2}$. The results achieved using novel single sine trigonometric-valued neutrosophic-weighted aggregation operators were the same as the results demonstrate existing techniques. Therefore, this study proposed the list of novel sine trigonometric aggregation operators to aggregate the single-valued neutrosophic information more effectively and efficiently. Using the proposed sine trigonometric aggregation operators, we sound the best alternative out of a collection of alternatives given by the decision-maker. Therefore, the proposed decision-making methodology based on sine trigonometric operational rules, helps us to find the best solution in decision-support systems as applications.

\section{Conclusions}

The process of industrialization has significantly increased energy consumption throughout the world. The objective of the proposed research is to present a novel decision-making approach for the selection of hydrogen power plant sites. To accomplish this task, novel sine trigonometric function-based operational laws are introduced under SVNNs. Utilizing these STOLs proposed some aggregation operators, namely, sine trigonometric SVN weighted averaging/geometric aggregation operators and sine trigonometric SVN-ordered weighted averaging/geometric aggregation operators. The various fundamental relations between the developed AOs are studied and presented in details. To implement the proposed laws on to the DMPs, we designed a new MADM algorithm with decision-making problems where the preferences are assessed in terms of SVNNs. The utilized single-valued neutrosophic information measures have been found to be significantly efficient to handle the uncertainty in decision-making problems. The functionality of the developed method are tested over the illustrated example of hydrogen power plant site selection and superiority as well as feasibility of the method are examined in details. A comparative analysis with several existing works are also done to check its performance.

In the future research, the method proposed in this paper will be applied to other uncertain fields, such as probabilistic linguistic term sets, interval-value SVNSs, and so on. Besides, the proposed method can be applied to other areas, such as medical health diagnosis, green supplier selection, and so on.

Author Contributions: All authors contributed equally to this work. All authors have read and agreed to the published version of the manuscript.

Funding: This paper was supported by Major Humanities and Social Sciences Research Projects in Zhejiang Universities (No. 2018QN058), China Postdoctoral Science Foundation (No. 2019M651403), Zhejiang Province Natural Science Foundation (No. LY18G010007; No. LQ20G010001), Ningbo Natural Science Foundation (No. 2019A610037), and First Class Discipline of Zhejiang - A (Zhejiang Gongshang University - Statistics).

Acknowledgments: The authors 1 \& 2 extend their appreciation to the Higher Education Commission, Pakistan under National Research Program for Universities (NRPU), Project no. 10701/KPK/ NRPU/R\&D/HEC/2017. The authors $3 \& 4$ work under the Major Humanities and Social Sciences Research Projects in Zhejiang Universities (No. 2018QN058), China Postdoctoral Science Foundation (No. 2019M651403), Zhejiang Province Natural Science Foundation (No. LY18G010007; No. LQ20G010001), Ningbo Natural Science Foundation (No. 2019A610037), and First Class Discipline of Zhejiang - A (Zhejiang Gongshang University - Statistics).

Conflicts of Interest: The authors declare no conflicts of interest. 


\section{References}

1. Pilavachi, P.A.; Stephanidis, S.D.; Pappas, V.A.; Afgan, N.H. Multi-criteria evaluation of hydrogen and natural gas fuelled power plant technologies. Appl. Therm. Eng. 2009, 29, 2228-2234. [CrossRef]

2. Hennicke, P.; Fischedick, M. Towards sustainable energy systems: The related role of hydrogen. Energy Policy 2006, 34, 1260-1270. [CrossRef]

3. Gökalp, I.; Lebas, E. Alternative fuels for industrial gas turbines (AFTUR). Appl. Therm. Eng. 2004, 24, 1655-1663. [CrossRef]

4. Juste G. Hydrogen injection as additional fuel in gas turbine combustor. Evaluation of effects. Int. J. Hydrogen Energy 2006, 31, 2112-2121. [CrossRef]

5. Zadeh, L.A. Fuzzy sets. Inf. Control. 1965, 8, 338-353. [CrossRef]

6. Chen, S.M.; Tan, J.M. Handling multicriteria fuzzy decision-making problems based on vague set theory. Fuzzy Sets Syst. 1994, 67, 163-172. [CrossRef]

7. Bellman, R.E.; Zadeh, L.A. Decision-making in a fuzzy environment. Manag. Sci. 1970, 17, B-141. [CrossRef]

8. Atanassov, K.T. Intuitionistic fuzzy sets. Fuzzy Sets Syst. 1986, 20, 87-96. [CrossRef]

9. Xu, Z. Intuitionistic fuzzy aggregation operators. IEEE Trans. Fuzzy Syst. 2007, 15, 1179-1187.

10. Xu, Z.; Yager, R.R. Some geometric aggregation operators based on intuitionistic fuzzy sets. Int. J. Ofgeneral Syst. 2006, 35, 417-433. [CrossRef]

11. Zhao, H.; Xu, Z.; Ni, M.; Liu, S. Generalized aggregation operators for intuitionistic fuzzy sets. Int. J. Intell. Syst. 2010, 25, 1-30. [CrossRef]

12. Yager, R.R. Generalized orthopair fuzzy sets. IEEE Trans. Fuzzy Syst. 2016, 25, 1222-1230. [CrossRef]

13. Ali, M.I. Another view on q-rung orthopair fuzzy sets. Int. J. Intell. Syst. 2018, 33, 2139-2153. [CrossRef]

14. Hussain, A.; Irfan Ali, M.; Mahmood, T. Covering based q-rung orthopair fuzzy rough set model hybrid with TOPSIS for multi-attribute decision-making. J. Intell. Fuzzy Syst. 2019, 37, 981-993. [CrossRef]

15. Peng, X.; Dai, J.; Garg, H. Exponential operation and aggregation operator for q-rung orthopair fuzzy set and their decision-making method with a new score function. Int. J. Intell. Syst. 2018, 33, 2255-2282. [CrossRef]

16. Liu, P.; Wang, P. Multiple-attribute decision-making based on Archimedean Bonferroni Operators of q-rung orthopair fuzzy numbers. IEEE Trans. Fuzzy Syst. 2018, 27, 834-848. [CrossRef]

17. Liu, P.; Chen, S.M.; Wang, P. Multiple-attribute group decision-making based on q-rung orthopair fuzzy power maclaurin symmetric mean operators. IEEE Trans. Syst. Man Cybern. Syst. 2018. [CrossRef]

18. Khan, A.A.; Ashraf, S.; Abdullah, S.; Qiyas, M.; Luo, J.; Khan, S.U. Pythagorean fuzzy Dombi aggregation operators and their application in decision support system. Symmetry 2019, 11, 383. [CrossRef]

19. Cuong, B.C.; Kreinovich, V. Picture Fuzzy Sets-a new concept for computational intelligence problems. In Proceedings of the 2013 Third World Congress on Information and Communication Technologies (WICT 2013), Hanoi, Vietnam, 15-18 December 2013.

20. Ashraf, S.; Mahmood, T.; Abdullah, S.; Khan, Q. Different approaches to multi-criteria group decision-making problems for picture fuzzy environment. Bull. Braz. Math. Soc. 2019, 50, 373-397. [CrossRef]

21. Khan, S.; Abdullah, S.; Ashraf, S. Picture fuzzy aggregation information based on Einstein operations and their application in decision-making. Math. Sci. 2019, 13, 213-229. [CrossRef]

22. Zeng, S.; Asharf, S.; Arif, M.; Abdullah, S. Application of exponential jensen picture fuzzy divergence measure in multi-criteria group decision-making. Mathematics 2019, 7, 191. [CrossRef]

23. Khan, M.J.; Kumam, P.; Ashraf, S.; Kumam, W. Generalized Picture Fuzzy Soft Sets and Their Application in Decision Support Systems. Symmetry 2019, 11, 415. [CrossRef]

24. Khan, S.; Abdullah, S.; Abdullah, L.; Ashraf, S. Logarithmic Aggregation Operators of Picture Fuzzy Numbers for Multi-Attribute Decision-Making Problems. Mathematics 2019, 7, 608. [CrossRef]

25. Qiyas, M.; Abdullah, S.; Ashraf, S.; Abdullah, L. Linguistic Picture Fuzzy Dombi Aggregation Operators and Their Application in Multiple Attribute Group Decision-Making Problem. Mathematics 2019, 7, 764. [CrossRef]

26. Ashraf, S.; Abdullah, S.; Mahmood, T.; Aslam, M. Cleaner Production Evaluation in Gold Mines Using Novel Distance Measure Method with Cubic Picture Fuzzy Numbers. Int. J. Fuzzy Syst. 2019, 21, 2448-2461. [CrossRef]

27. Qiyas, M.; Abdullah, S.; Ashraf, S.; Aslam, M. Utilizing Linguistic Picture Fuzzy Aggregation Operators for Multiple-Attribute Decision-Making Problems. Int. J. Fuzzy Syst. 2020, 22, 310-320. [CrossRef] 
28. Ashraf, S.; Abdullah, S. Spherical aggregation operators and their application in multiattribute group decision-making. Int. J. Intell. Syst. 2019, 34, 493-523. [CrossRef]

29. Ashraf, S.; Abdullah, S.; Mahmood, T.; Ghani, F.; Mahmood, T. Spherical fuzzy sets and their applications in multi-attribute decision-making problems. J. Intell. Fuzzy Syst. 2019, 36, 2829-2844. [CrossRef]

30. Ashraf, S.; Abdullah, S.; Mahmood, T. Spherical fuzzy Dombi aggregation operators and their application in group decision making problems. J. Ambient Intell. Human. Comput. 2019, 1-19. [CrossRef]

31. Jin, Y.; Ashraf, S.; Abdullah, S. Spherical Fuzzy Logarithmic Aggregation Operators Based on Entropy and Their Application in Decision Support Systems. Entropy 2019, 21, 628. [CrossRef]

32. Jin, H.; Ashraf, S.; Abdullah, S.; Qiyas, M.; Bano, M.; Zeng, S. Linguistic Spherical Fuzzy Aggregation Operators and Their Applications in Multi-Attribute Decision-Making Problems. Mathematics 2019, 7, 413. [CrossRef]

33. Rafiq, M.; Ashraf, S.; Abdullah, S.; Mahmood, T.; Muhammad, S. The cosine similarity measures of spherical fuzzy sets and their applications in decision-making. J. Intell. Fuzzy Syst. 2019, 36, 6059-6073. [CrossRef]

34. Ashraf, S.; Abdullah, S.; Abdullah, L. Child Development Influence Environmental Factors Determined Using Spherical Fuzzy Distance Measures. Mathematics 2019, 7, 661. [CrossRef]

35. Ashraf, S.; Abdullah, S.; Aslam, M.; Qiyas, M.; Kutbi, M.A. Spherical fuzzy sets and its representation of spherical fuzzy t-norms and t-conorms. J. Intell. Fuzzy Syst. 2019, 36, 6089-6102. [CrossRef]

36. Zeng, S.; Hussain, A.; Mahmood, T.; Irfan Ali, M.; Ashraf, S.; Munir, M. Covering-Based Spherical Fuzzy Rough Set Model Hybrid with TOPSIS for Multi-Attribute Decision-Making. Symmetry 2019, 11, 547. [CrossRef]

37. Ashraf, S.; Abdullah, S.; Mahmood, T. GRA method based on spherical linguistic fuzzy Choquet integral environment and its application in multi-attribute decision-making problems. Math. Sci. 2018, 12, 263-275. [CrossRef]

38. Smarandache, F. A Unifying Field in Logics. Neutrosophy: Neutrosophic Probability, Set and Logic; American Research Press: Rehoboth, DE, USA, 1999.

39. Wang, H.; Smarandache, F.; Zhang, Y.Q.; Sunderraman, R. Single valued neutrosophic sets. Multispace Multistructure 2010, 4, 410-413.

40. Ye, J. Multicriteria decision-making method using the correlation coefficient under single-valued neutrosophic environment. Int. J. Gener. Syst. 2013, 42, 386-394. [CrossRef]

41. Liu, P.; Khan, Q.; Mahmood, T.; Smarandache, F.; Li, Y. Multiple attribute group decision-making based on 2-tuple linguistic neutrosophic Dombi power Heronian mean operators. IEEE Access 2019, 7, 100205-100230. [CrossRef]

42. Liu, P.; Khan, Q.; Mahmood, T. Some single-valued neutrosophic power muirhead mean operators and their application to group decision-making. J. Intell. Fuzzy Syst. 2019, 37, 2515-2537. [CrossRef]

43. Liu, P.; Mahmood, T.; Khan, Q. Group decision making based on power Heronian aggregation operators under linguistic neutrosophic environment. Int. J. Fuzzy Syst. 2018, 20, 970-985. [CrossRef]

44. Liu, P.; Khan, Q.; Ye, J.; Mahmood, T. Group decision-making method under hesitant interval neutrosophic uncertain linguistic environment. Int. J. Fuzzy Syst. 2018, 20, 2337-2353. [CrossRef]

45. Ye, J. Single valued neutrosophic cross-entropy for multicriteria decision-making problems. Appl. Math. Model. 2014, 38, 1170-1175. [CrossRef]

46. Liu, P. The aggregation operators based on archimedean $\mathrm{t}$-conorm and t-norm for single-valued neutrosophic numbers and their application to decision-making. Int. J. Fuzzy Syst. 2016, 18, 849-863. [CrossRef]

47. Ji, P.; Wang, J.Q.; Zhang, H.Y. Frank prioritized Bonferroni mean operator with single-valued neutrosophic sets and its application in selecting third-party logistics providers. Neural Comput. Appl. 2018, 30, 799-823. [CrossRef]

48. Liu, P.; Liu, X. The neutrosophic number generalized weighted power averaging operator and its application in multiple attribute group decision-making. Int. J. Mach. Learn. Cybern. 2018, 9, 347-358. [CrossRef]

49. Semenas, R.; Bausys, R. Modelling of Autonomous Search and Rescue Missions by Interval-Valued Neutrosophic WASPAS Framework. Symmetry 2020, 12, 162. [CrossRef]

50. Cao, C.; Zeng, S.; Luo, D. A single-valued neutrosophic linguistic combined weighted distance measure and its application in multiple-attribute group decision-making. Symmetry 2019, 11, 275. [CrossRef] 
51. Zhao, S.; Wang, D.; Liang, C.; Leng, Y.; Xu, J. Some Single-Valued Neutrosophic Power Heronian Aggregation Operators and Their Application to Multiple-Attribute Group Decision-Making. Symmetry 2019, 11, 653. [CrossRef]

52. Shao, S.; Zhang, X.; Zhao, Q. Multi-attribute decision-making based on probabilistic neutrosophic hesitant fuzzy Choquet aggregation operators. Symmetry 2019, 11, 623. [CrossRef]

53. Yu, L.P.; Zeng, S.Z.; Merigo, J.M.; Zhang, C.H. A new distance measure based on the weighted induced method and its application to Pythagorean fuzzy multiple attribute group decision-making. Int. J. Intell. Syst. 2019, 34, 1440-1454 [CrossRef]

54. Ye, J. Exponential operations and aggregation operators of interval neutrosophic sets and their decision-making methods. SpringerPlus 2016, 5, 1488. [CrossRef] [PubMed]

55. Garg, H. New logarithmic operational laws and their applications to multiattribute decision-making for single-valued neutrosophic numbers. Cogn. Syst. Res. 2018, 52, 931-946. [CrossRef]

56. Ashraf, S.; Abdullah, S.; Smarandache, F. Logarithmic Hybrid Aggregation Operators Based on Single Valued Neutrosophic Sets and Their Applications in Decision Support Systems. Symmetry 2019, 11, 364. [CrossRef]

57. Ye, J. Subtraction and division operations of simplified neutrosophic sets. Information 2017, 8, 51. [CrossRef]

58. Zhang, X.; Bo, C.; Smarandache, F.; Dai, J. New inclusion relation of neutrosophic sets with applications and related lattice structure. Int. J. Mach. Learn. Cybern. 2018, 9, 1753-1763. [CrossRef]

59. Zeng, S.Z.; Su, W.H.; Zhang, C.H. Intuitionistic fuzzy generalized probabilistic ordered weighted averaging operator and its application to group decision making. Technol. Econ. Dev. Econ. 2016, 22, 177-193. [CrossRef]

60. Zeng, S. Pythagorean fuzzy multiattribute group decision-making with probabilistic information and OWA approach. Int. J. Intell. Syst. 2017, 32, 1136-1150. [CrossRef]

61. Zhang, C.H.; Chen, C.; Streimikiene, D.; Balezentis, T. Intuitionistic fuzzy MULTIMOORA approach for multi-criteria assessment of the energy storage technologies. Appl. Soft Comput. 2019, 79, 410-423. [CrossRef]

62. Zeng, S.; Chen, S.M.; Kuo, L.W. Multiattribute decision-making based on novel score function of intuitionistic fuzzy values and modified VIKOR method. Inf. Sci. 2019, 488, 76-92. [CrossRef]

63. Zeng, S.Z.; Peng, X.M.; Baležentis, T.; Streimikiene, D. Prioritization of low-carbon suppliers based on Pythagorean fuzzy group decision-making with self-confidence level. Econ. Res.-Ekon. Istraživanja 2019, 32, 1073-1087. [CrossRef]

64. Peng, J.J.; Wang, J.Q.; Wang, J.; Zhang, H.Y.; Chen, X.H. Simplified neutrosophic sets and their applications in multi-criteria group decision-making problems. Int. J. Syst. Sci. 2016, 47, 2342-2358. [CrossRef]

65. Garg, H. Novel single-valued neutrosophic aggregated operators under Frank norm operation and its application to decision-making process. Int. J. Uncertain. Quantif. 2016, 6, 361-375.

66. Liu, P.; Chu, Y.; Li, Y.; Chen, Y. Some Generalized Neutrosophic Number Hamacher Aggregation Operators and Their Application to Group Decision-Making. Int. J. Fuzzy Syst. 2014, 16, 242-255. 